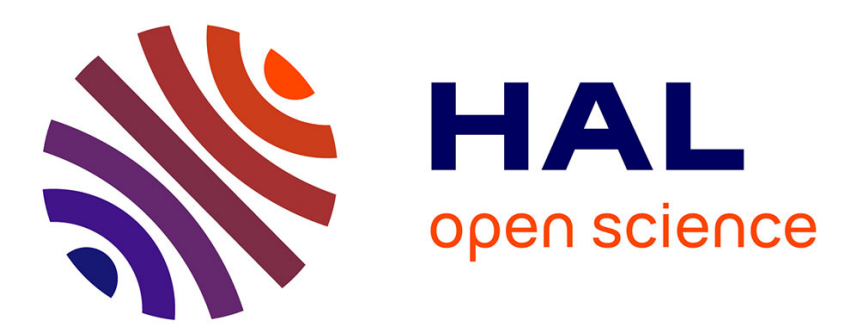

\title{
Nonlinear dynamics of two helical vortices: A dynamical system approach
}

\author{
Ivan Delbende, Can Selçuk, Maurice Rossi
}

\section{To cite this version:}

Ivan Delbende, Can Selçuk, Maurice Rossi. Nonlinear dynamics of two helical vortices: A dynamical system approach. Physical Review Fluids, 2021, 6 (8), 10.1103/PhysRevFluids.6.084701 . hal03452752

\section{HAL Id: hal-03452752 \\ https://hal.science/hal-03452752}

Submitted on 29 Nov 2021

HAL is a multi-disciplinary open access archive for the deposit and dissemination of scientific research documents, whether they are published or not. The documents may come from teaching and research institutions in France or abroad, or from public or private research centers.
L'archive ouverte pluridisciplinaire HAL, est destinée au dépôt et à la diffusion de documents scientifiques de niveau recherche, publiés ou non, émanant des établissements d'enseignement et de recherche français ou étrangers, des laboratoires publics ou privés. 


\title{
Nonlinear Dynamics of Two Helical Vortices: a Dynamical System Approach
}

\author{
Ivan Delbende* and Can Selçuk \\ Sorbonne Université, UFR d'Ingénierie, 4 place Jussieu, 75005 Paris, France and \\ LIMSI, CNRS, Université Paris-Saclay, rue du Belvédère, 91405 Orsay, France \\ Maurice Rossi \\ CNRS, UMR 7190, Institut Jean Le Rond d'Alembert, 75005 Paris, France and \\ Sorbonne Université, UMR 7190, Institut Jean Le Rond d'Alembert, 75005 Paris, France
}

(Dated: June 29, 2021)

\begin{abstract}
The paper investigates the variety of dynamics displayed by two identical interwoven helical vortices for different values of their helical pitch. We present simplified approaches aimed at understanding their nonlinear time evolution: in order of increasing complexity, we use models of nonlinear interaction of two point-vortex alleys, of two vortex-ring filament alleys, and of two inviscid helical filaments. Each of these inviscid models leads to a dynamical system of few degrees of freedom that can be analyzed in terms of orbits in a phase space structured by elliptical and hyperbolic points. At low pitch, the basic state with the two helical vortices in symmetric configuration is an unstable equilibrium, and corresponds to hyperbolic points in the phase space. An initial perturbation induces different types of dynamics depending on its direction and amplitude. Features observed for increasing helical pitches in direct numerical simulations or in experiments are gradually understood as the model complexity is increased. At small pitch, leapfrog cycles are triggered by axial or azimuthal displacements of the vortices, while radial perturbations trigger overtaking; for both regimes, a periodic change in the interwaving order of the vortices occurs. Above a critical pitch value, small amplitude perturbations trigger a new regime called fluttering, mainly characterized by a periodic evolution of the vortex radii without change of the interwaving order; large amplitude displacements then still allow for leapfrog dynamics far from equilibrium. The amplitudes of the radial excursions of the vortices induced by small perturbations are characterized over the whole range of helical pitches, up to the linear stabilisation threshold. Mimicking viscous effects in the point-vortex alley model sheds some light on how dissipation causes overtaking to turn into leapfrog (the reverse is not observed).
\end{abstract}

\footnotetext{
* Email: ivan.delbende@ sorbonne-universite.fr; now at Sorbonne Université, UMR 7190, Institut Jean Le Rond d'Alembert, 75005 Paris, France
} 


\section{INTRODUCTION}

The wake behind helicopters rotors, helices or wind/marine turbines are characterized by several interwoven tip vortices, each one being produced by a single blade. These structures interact in a non trivial way, which eventually leads to wake destructuration. In order to understand such complex flows, it is pertinent to study the dynamics once the tip vortices are formed: indeed, the near wake made of $N$ identical tip vortices can be used as a base flow [7, 16, 21, 23]. The dynamics can then be analyzed in terms of linear and nonlinear evolution of perturbations evolving on top of this base flow. Such perturbations can be due to rotor asymmetry which displaces the vortices from their ideal location. This effect could also be used as a means of manipulating and controlling the wake. In other circumstances, e.g. for floating wind turbines, the oscillating motion of the rotor induces long-wave perturbations on the vortices, which strongly affect the wake dynamics. Getting information on how different perturbations can induce different types of vortex trajectories, and possibly different wake dynamics, is thus of great practical interest.

When one neglects its slight downstream expansion, the near wake satisfies a symmetry that simplifies the study: helical symmetry. This means that the flow remains invariant through a translation of arbitrary magnitude $H$ along the rotor $z$-axis coupled with a rotation of angle $\theta_{s}=H / L$ around the same axis. Parameter $L$ is fixed and imposes the pitch $\ell=2 \pi L$, that is the spatial periodicity of one helix along the $z$-axis. A related parameter is the reduced pitch $L / R$, defined as the pitch $\ell$ divided by the circumference $2 \pi R$ of the rotor. This parameter depends on the angular velocity, blade geometry and the upstream velocity.

For a single helical vortex (one blade helix), such helically symmetric base flow can be easily generated and the linear evolution of long-wave perturbations super-imposed on it can be studied in the framework of theory [29] as well as numerics [5, 28] and physical experiments [20]. The most unstable perturbations do not affect the internal structure of the single vortex but displace its core as a whole so that successive turns are arranged in pairs. This geometric change is similar to what is observed for the pairing process which occurs in an alley of regularly spaced two-dimensional vortices. This analogy is actually dynamically based: the linear process could be indeed related more quantitatively to the phenomenon of vortex pairing [20].

For two identical interwoven helical vortices, a similar process is at work, as shown by theoretical studies [18, 19], direct numerical simulations (DNS) [25] and water channel experiments [12, 21]. At low pitch, the most amplified linear mode is again analogous to the pairing instability mode of an alley of regularly spaced two-dimensional point vortices. Contrary to the case of a single helical vortex however, this mode is helically symmetric with the same pitch. This implies that during the three-dimensional nonlinear evolution, the helical symmetry is preserved. The present study relies on this property and addresses the nonlinear dynamics of two identical helical vortices in the framework of helical symmetry, run at fixed helical pitch. The pitch can be varied from small to large values, reaching configurations that cannot be easily investigated due to space limitation in water or wind channels. The purpose of the present work is (i) to extend into the nonlinear regime the inviscid analogy previously shown between linear instability of helical vortices and point-vortex alleys instability, and (ii) to provide dynamical systems of few degrees of freedom in which the distinct mechanisms observed in the dynamics of two helical vortices with same circulations and pitches can be accounted for.

The linear stability of one alley of regularly spaced point vortices [3, 13] and the examination of the dominant eigenmode unveils the way the system separates into two sub-alleys, each containing every two vortices, traveling in opposite directions at 45 degrees with respect to the initial alley axis. The nonlinear dynamics of these periodic sub-alleys have been considered by Stremler [27]. This author used a Hamiltonian system viewpoint as well as conservation laws, and showed, among others, the possibility of bounded and 
unbounded trajectories. The present study gives an important field of application to such investigations, namely that of helical tip vortices, as each sub-alley can model one helical vortex.

Some authors have also used a more refined analogy between helical vortex and a system of vortex rings, thus taking core size and vortex curvature effects into account. This has been performed to describe the spatial development of a wind-turbine wake [4] or to investigate temporal and spatio-temporal instability properties of a set of helical vortices, replaced by a regular array of coaxial vortex rings [6]: the temporal linear stability properties of such an infinite array, first considered by Levy \& Forsdyke [14], were found qualitatively close to those for a point-vortex alley provided the reduced pitch and the core-to-helix radius ratio be sufficiently small. In the present paper, we extend the temporal study into the nonlinear regime by considering two periodic alleys of vortex rings, each describing one helical vortex, using a Hamiltonian formulation for coaxial vortex rings [17]. The well-known leapfrog phenomenon of vortex rings has been extensively studied, but it generally involves a few vortices, typically 2 or 3 [8]. It is the first time, to our knowledge, that a nonlinear study is performed on the nonlinear dynamics of two infinite vortex-ring arrays, viewed here as a pertinent model for helical vortex dynamics.

When torsion effects cannot be neglected (typically for values of the reduced pitch $L / R$ larger than 0.3 ), the full geometry of helical vortices has to be taken into account: we then use a model based on a vortexfilament approach in the cut-off approximation [9]. A Hamiltonian formulation is too much involved in this latter case, but the paper endeavors to present the results in the same form as for simplified models where the Hamiltonian formulation is more easily tractable.

The structure of the paper is as follows. The nonlinear dynamics of two infinite periodic alleys of point vortices is presented in section II. Vortex curvature and core size are introduced in section III which describes the dynamics of two infinite alleys of inviscid vortex rings. Section IV additionally includes vortex torsion and presents the dynamics of two helical vortex filaments in the cut-off approximation. Such approach is found necessary to describe the bifurcations and the variety of dynamical regimes at large pitch. In section $\mathrm{V}$, it is shown that dissipation effects can be mimicked within the point-vortex theory. The detailed derivation of dynamical systems is given for point-vortex and vortex-ring alleys in Appendix A, and for helical filaments in Appendix B.

\section{TWO HELICAL VORTICES AS TWO PERIODIC ALLEYS OF POINT VORTICES}

Helical flows of pitch $\ell=2 \pi L$ along the $z$-axis have been defined in the introduction. Using the unit vector $\boldsymbol{e}_{z}$ along the $z$-axis and an associated polar basis $\boldsymbol{e}_{r}(\theta), \boldsymbol{e}_{\theta}(\theta) \equiv \boldsymbol{e}_{z} \times \boldsymbol{e}_{r}$ in the orthogonal plane $z=0$, a helically symmetric velocity field $\boldsymbol{u}(r, \boldsymbol{\theta}, z, t)$ can be expressed as follows:

$$
\boldsymbol{u}=u_{r}(r, \varphi, t) \boldsymbol{e}_{r}(\theta)+u_{\theta}(r, \varphi, t) \boldsymbol{e}_{\theta}(\theta)+u_{z}(r, \varphi, t) \boldsymbol{e}_{z}, \quad \text { where } \varphi \equiv \theta-\frac{z}{L}
$$

A positive pitch $\ell>0$ corresponds to a right-handed helix and a negative pitch $\ell<0$ to a left-handed one. If initially a flow is helically symmetric, it remains so in the framework of Navier-Stokes or Euler dynamics in an unbounded domain (see [10] for more details). Within this framework, we study nonlinear dynamics related to a base flow defined by two helical vortices of same core size $a_{0}$, circulation $\Gamma$ and distance $R_{0}$ from the symmetry axis. The two vortices are separated along the axis by a distance $\ell / 2$ (see figure 1a). The internal structure of this state forms a Navier-Stokes quasi-equilibrium [24] as well as an Euler equilibrium, a meridional section of which is illustrated in figure $1 \mathrm{~b}$. This base flow is adequate to study two-blade turbine 
(a)

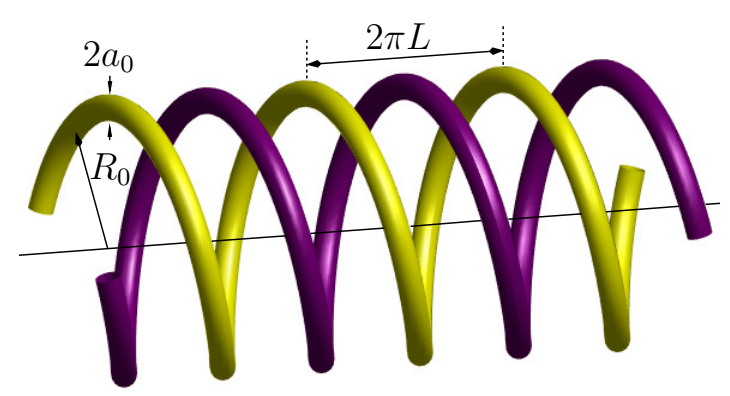

(b)

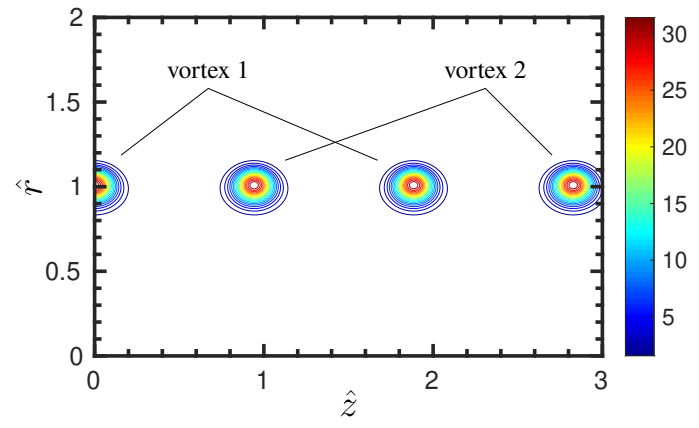

FIG. 1. (a) A typical helically symmetric base flow with two helical vortices. (b) A helically symmetric quasiequilibrium solution obtained numerically: meridional half-plane section displaying isolines of the helical vorticity component, for two vortices with identical circulation, radial position, core size $\hat{a}_{0}=a_{0} / R_{0}=0.1$ and reduced pitch $\hat{L}=L / R_{0}=0.3$.

or propeller rotor wakes when focusing on the dynamics of tip vortices. We hereafter use dimensionless variables based on length scale $R_{0}$ and velocity scale $\Gamma / R_{0}$. Dimensionless variables will be written with a hat, except when there is no ambiguity. The dimensionless parameters are the reduced pitch $\hat{L}=L / R_{0}$ and ratio $\hat{a}_{0}=a_{0} / R_{0}$. As already mentioned in the introduction, this base state is unstable except at large pitch: the most unstable linear mode does not break the helical symmetry and the total perturbed flow remains helically symmetric during the subsequent time evolution [25]. The purpose of the present work is to study the nonlinear evolution following the linear phase of exponential growth from various theoretical model standpoints. We investigate initial perturbations that may be different from the most amplified mode, and not necessarily of small amplitude, but always within the helically symmetric framework. In particular, we analyze perturbations such as radial or axial shifts of arbitrary amplitude which are closer to experimental achievements: vortices can be manipulated by static modifications of the rotor. For instance, offsetting a two-blade rotor along the direction of the chordlines leads to a change of the radial locations of the two tip vortices. The offset can also be done in the direction normal to the chordlines, allowing for a change in the azimuthal locations, so that one vortex is no more opposite to its companion. This latter perturbation is equivalent to a relative shift of the vortices in the axial direction. All these static perturbations lead to helically symmetric flows. Each helical vortex indexed by an integer $p$ (where $p=1$ or 2) can be described by its core size $a_{p}(t)$ and the spatial location of its centerline

$$
\boldsymbol{R}_{p}(z, t)=r_{p}(t) \boldsymbol{e}_{r}\left(\varphi_{p}(t)+\frac{z}{L}\right)+z \boldsymbol{e}_{z},
$$

where parameter $z$ is continuously varied. This line is completely determined by its intersection with the plane $z=0$ provided by the radial position $r_{p}(t)$ i.e. the distance of its vortex center from the symmetry axis and an azimuthal angle $\varphi_{p}(t)$. This helical line has a radius of curvature $\left(r_{p}^{2}+L^{2}\right) / r_{p}$ and a radius of torsion $\left(r_{p}^{2}+L^{2}\right) / L$. For the basic state, $r_{p}=R_{0}$ remains constant so that the reduced pitch $\hat{L}$ is nothing but the ratio between radius of curvature and radius of torsion.

In a first bold move, we consider the case when the radius of torsion is much larger than the radius of curvature, i.e. $\hat{L} \ll 1$, and the radius of curvature itself is much larger than the core size, i.e. $a_{0} / R_{0}=\hat{a}_{0} \ll$ $\hat{L} \ll 1$. In this framework, the two helical vortices are replaced by their intersections with the meridional 
(a)

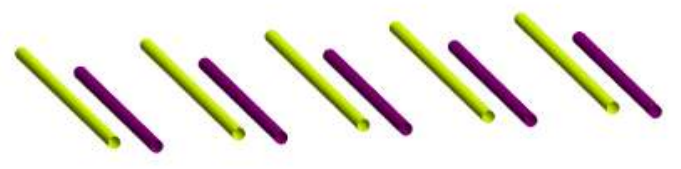

(b)

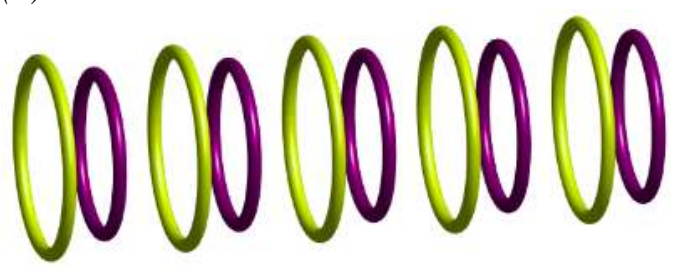

FIG. 2. Approximation of two helical vortices: (a) Two periodic alleys of point vortices. (b) Two periodic alleys of vortex ring filaments.

plane, resulting in two infinite spatially-periodic alleys of vortices such as represented in figure $1 \mathrm{~b}$. As here only two-dimensional interactions are taken into account, the system reduces to two $2 \mathrm{D}$ vortex alleys (see figure 2a), which can be further simplified by reducing vortices to point vortices of identical circulation $\Gamma$. Let $x$ denote the axis containing all vortices of the base state, and $y$ the orthogonal direction. Each helical vortex labelled by index $p=1$ or 2 corresponds to an infinite periodic alley of point vortices of circulation $\Gamma$ located at $\left[x_{p}(t)+k \ell, y_{p}(t)\right]$, where $k \in \mathbb{Z}$ and $\left[x_{p}(t), y_{p}(t)\right]$ stands for the coordinates of one intersection selected at initial time and thereafter followed. The locations $\left[x_{i}(t), y_{i}(t)\right]$ of the two infinite point-vortex alleys are governed (see appendix A) by a four-dimensional dynamical system

$$
\frac{\mathrm{d}}{\mathrm{d} t}\left(\begin{array}{c}
x_{i} \\
y_{i}
\end{array}\right)=\frac{\Gamma}{2 \ell} \sum_{\substack{p=1 \\
p \neq i}}^{2} \frac{1}{\cosh \left[2 \pi\left(y_{i}-y_{p}\right) / \ell\right]-\cos \left[2 \pi\left(x_{i}-x_{p}\right) / \ell\right]}\left(\begin{array}{c}
-\sinh \left[2 \pi\left(y_{i}-y_{p}\right) / \ell\right] \\
\sin \left[2 \pi\left(x_{i}-x_{p}\right) / \ell\right]
\end{array}\right), \quad i=1,2 .
$$

Because of momentum conservation (see appendix A), the two quantities $\frac{1}{2}\left(x_{1}+x_{2}\right)$ and $\frac{1}{2}\left(y_{1}+y_{2}\right)$ are invariant. For the steady base flow situation, $\left(x_{1}, y_{1}\right)=(0,0)$ and $\left(x_{2}, y_{2}\right)=\left(\frac{1}{2} \ell, 0\right)$. A perturbation of this base flow situation supposes that vortex 1 is shifted to $\left(x_{1}, y_{1}\right)=\frac{1}{2} \ell(\xi, \eta)$, where $(\xi, \eta)$ are dimensionless quantities. The momentum conservation then implies that vortex 2 , located at $\left(x_{2}, y_{2}\right) \equiv \frac{1}{2} \ell\left(\xi^{\prime}, \eta^{\prime}\right)$, should remain symmetric with respect to the point $\left(\frac{1}{4} \ell, 0\right)$, i.e. $\left(\xi^{\prime}, \eta^{\prime}\right)=(1-\xi,-\eta)$, as illustrated in figure $3 \mathrm{a}$. The problem then reduces to a two-dimensional Hamiltonian system

$$
\frac{\mathrm{d}}{\mathrm{d} t}\left(\begin{array}{l}
\xi \\
\eta
\end{array}\right)=\left(\begin{array}{c}
\partial \hat{H} / \partial \eta \\
-\partial \hat{H} / \partial \xi
\end{array}\right) \text { with } \hat{H}(\xi, \eta)=-\frac{1}{8 \pi} \log [\cosh (2 \pi \eta)+\cos (2 \pi \xi)]
$$

cast in dimensionless form using the time scale $\ell^{2} / \Gamma(\Gamma>0$ is assumed here, the case $\Gamma<0$ is equivalent).

Isolines of $\hat{H}$ in the $(\xi, \eta)$-plane are displayed in figure $3 \mathrm{~b}$ and correspond to vortex trajectories of both alley 1 and alley 2 (because of the above symmetry). The heteroclinic separatrices structure this twodimensional phase space dynamics: two types of evolutions are observed. The first case occurs when the initial perturbation moves alley 1 along the $\xi$-axis. As a consequence of the symmetry, the second alley is moved along the $\xi$-axis in the opposite direction (figure 3c), so that both vortices are located inside the heteroclinic separatrix. Over time, one alley is moving downwards then upwards, the other one upwards then downwards. After one cycle, alleys have switched their initial locations and thus exchange their roles, as shown in the time evolution of figure $4 a-4 b$. The second case occurs when the initial perturbation moves alley 1 along the $\eta$-axis. Because of the symmetry, the second alley is moved along the $\eta$-axis in the oppo- 
(a)
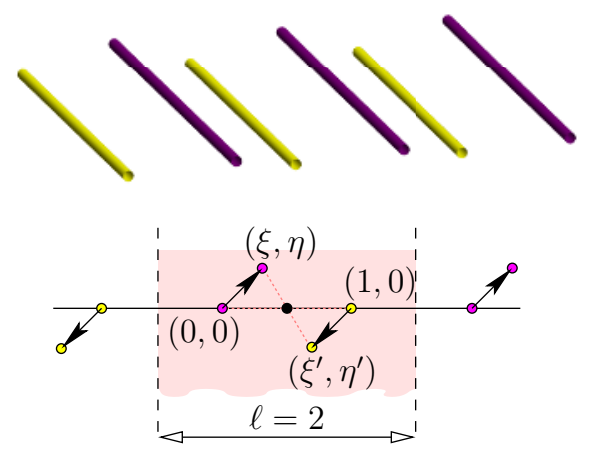

(c)

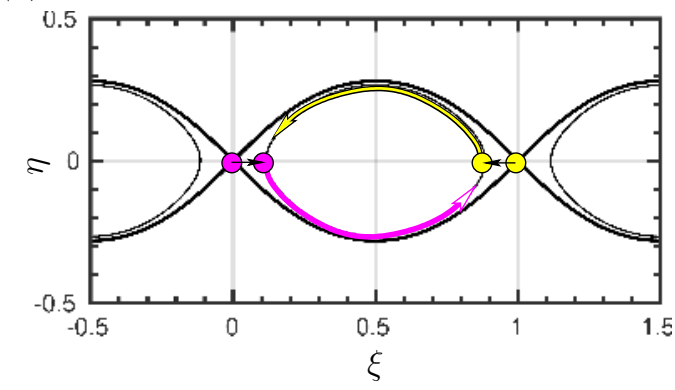

(b)

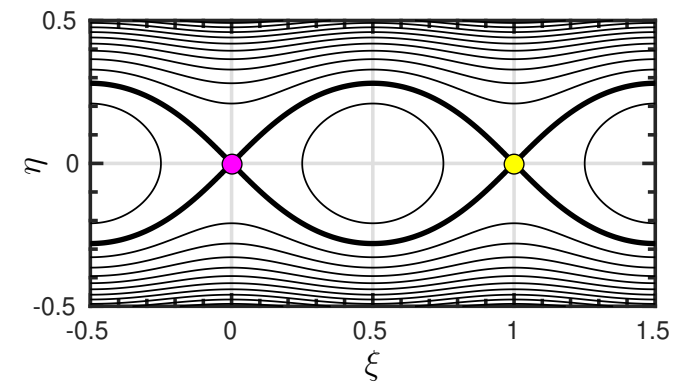

(d)

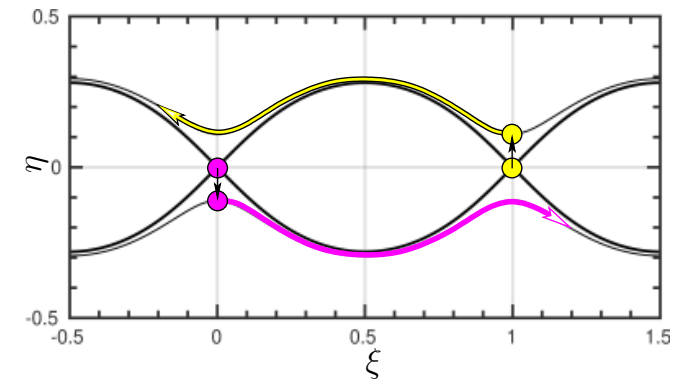

FIG. 3. Two alleys of straight vortices. (a) Top: typical perturbation of the base state in perspective view. Bottom: same perturbation in the $(\xi, \eta)$-plane. The locations of the two alleys are $(\xi, \eta)$ and $\left(\xi^{\prime}, \eta^{\prime}\right)=(1-\xi,-\eta)$. (b) Isolines $\hat{H}=$ cst in the phase plane $(\xi, \eta)$ (only one spatial period is represented); color points correspond to the base state. (c) Leapfrogging vortex trajectories obtained when vortices are initially perturbed along $\xi$. (d) Overtaking vortex trajectories obtained when vortices are initially perturbed along $\eta$.

site direction (see figure 3d). Both alleys are oscillating periodically but one remains always below the level of the base solution at $\eta=0$ while its companion remains always above, as shown in figure $4 \mathrm{c}-4 \mathrm{~d}$.

In terms of three-dimensional dynamics, the basic cycle (hereafter called exchange cycle) followed by the two point vortex alleys can be interpreted as one helical vortex going towards the axis and then back, while the companion vortex is going away from the axis and back. This pattern is also obtained by DNS (see figure 5) of two helical vortices within helical symmetry [25] or in the fully 3D setting [26]. It has also been quantified in an experimental two-blade rotor experiment by Quaranta et al. [21], moving downwards with the flow (see their figure 20a).

This simple model however predicts more: when shifted axially (along $\xi$ ) at $t=0$, the two alleys swap roles at each cycle. However, when shifted transversally (along $\eta$ ), one alley remains always below the other one and overtakes it periodically. In DNS, the first behaviour that recalls the leapfrog mechanism for vortex rings, is indeed observed when two helical vortices are initially moved axially; the second behaviour denominated as overtaking dynamics has been observed when the two helical vortices are initially moved radially [25]. In the experimental situation [21] however, one observes slightly more than one cycle because 
(a)

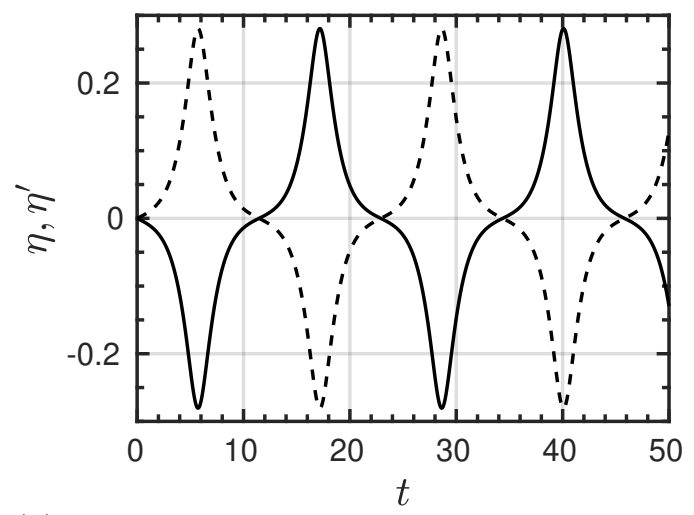

(c)

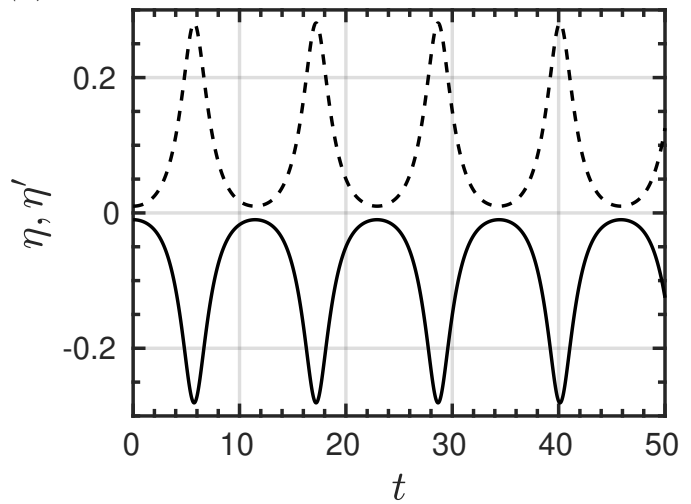

(b)

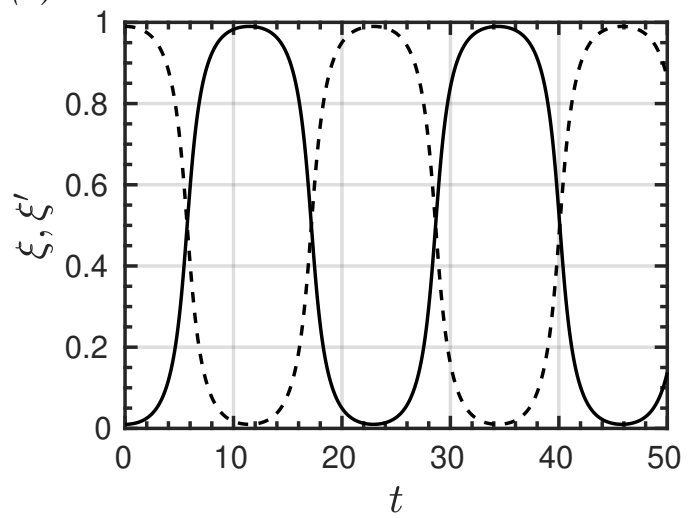

(d)

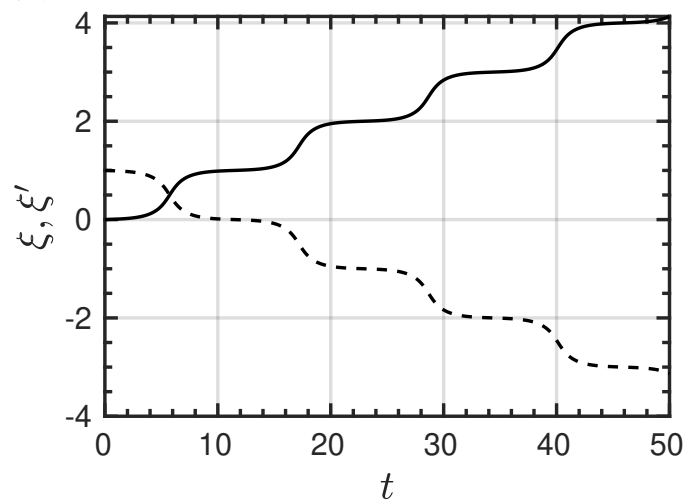

FIG. 4. Time integration of dynamical system (4) showing (a) and (c) the transverse, (b) and (d) the axial location of the two vortex alleys in time. The initial conditions are (a)-(b) an axial perturbation of amplitude $|\xi|=0.01$ or (c)(d) a transverse perturbation of amplitude $|\eta|=0.01$. Movies of the vortex motion presented simultaneously in 3D perspective and in the $(\xi, \eta)$-plane are provided in the Supplemental Material [1].

of the limitation in size of the set-up, but this is enough to evidence leapfrog although a transverse shift is applied to the vortices. This may be due to a dissipation effect able to turn overtaking into leapfrogging (see section $\mathrm{V}$ ).

\section{TWO HELICAL VORTICES AS TWO PERIODIC ALLEYS OF VORTEX RINGS}

The point-vortex model (4) does not reproduce certain features observed for helical vortices. For instance, trajectories going below or above the base flow level $\eta=0$ possess symmetric amplitudes in the pointvortex model (figure 4). The dimensionless amplitude being constant, its dimensional counterpart linearly increases with the pitch $\ell$ and is thus not bounded. For helical vortices however, amplitudes are necessarily bounded while increasing the pitch $\ell$. This point can be accounted for if the two helical vortices are now 

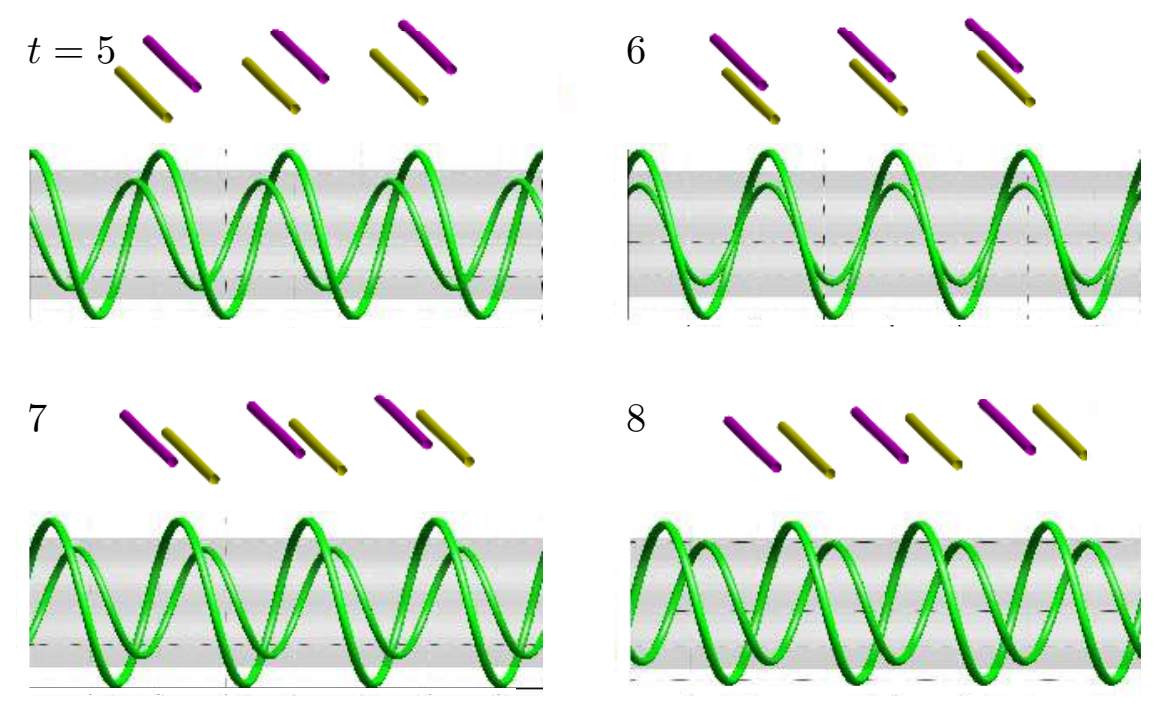

FIG. 5. Basic exchange cycle in the time evolution of two helical vortices obtained by DNS for initial core size $\hat{a}_{0}=0.1$ and $\hat{L}=0.3$; isosurfaces of vorticity at $\frac{1}{4}$ of the maximum level. Above each snapshot, analogous situations obtained via the two point-vortex alley model are represented, corresponding to times $t=5,6,7$ and 8 of figure 4 .

viewed as two infinite periodic alleys of coaxial rings with common circulations $\Gamma$ (figure $2 \mathrm{~b}$ ). In this second model, vortex torsion is not yet taken into account but curvature and core size are both introduced - the latter is compulsory as soon as curvature is nonzero to avoid the singular velocity of curved line vortices. This approach imposes the reduced pitch $\hat{L}=\ell /\left(2 \pi R_{0}\right)$ to be small but this condition is less stringent than for the first model. The theory is based on a filamentary approach for three-dimensional vortical flows, called the cut-off theory [22] which assumes the vortex core size $a_{p}$ to remain small compared to other characteristic lengths, namely to ring radius and pitch. Furthermore, the internal velocity field at leading order is assumed to be axisymmetric in a section orthogonal to the local vortex axis. Within this hypothesis, the flow dynamics is described by the time evolution of vortex lines. Each alley of rings is periodic of period $\ell$ along the common $z$-axis and has radius $r_{p}(t)$ and axial position $z_{p}(t)(p=1,2)$. Appendix A introduces the dynamical equations for two such alleys of vortex rings. They derive from a Hamiltonian $H$ corresponding to one spatial period $z \in\left[0, \ell\left[\right.\right.$, where only three degrees of freedom intervene: the radii $r_{1}$ and $r_{2}$ as well as the relative axial position $z_{1}-z_{2}$. In addition, the conservation of the momentum component $P_{z} \equiv \Gamma \pi\left[r_{1}^{2}+r_{2}^{2}\right]$ over one spatial period imposes a relation between $r_{1}$ and $r_{2}$. Here again, the dynamical system is thus two-dimensional.

The base state of figure $1 \mathrm{~b}$ is approximated by the two ring alleys axially shifted by $\ell / 2$, with the same radius $R_{0}$ and core size $a_{0}$. This base state is perturbed by moving alley 1 to radial position $r_{1} \equiv R_{0}(1+\eta)$ and alley 2 to $r_{2} \equiv R_{0}\left(1+\eta^{\prime}\right)$ and changing their relative axial position $z_{1}-z_{2} \equiv 2 R_{0} \zeta$. The Hamiltonian of the two alleys given by equation (A16) is written here in dimensionless form (rescaled using circulation $\Gamma$ 
(a)

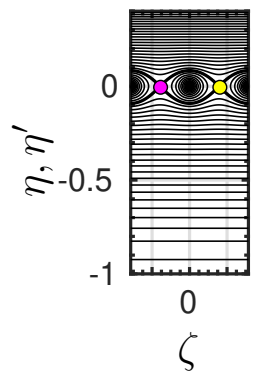

(b)

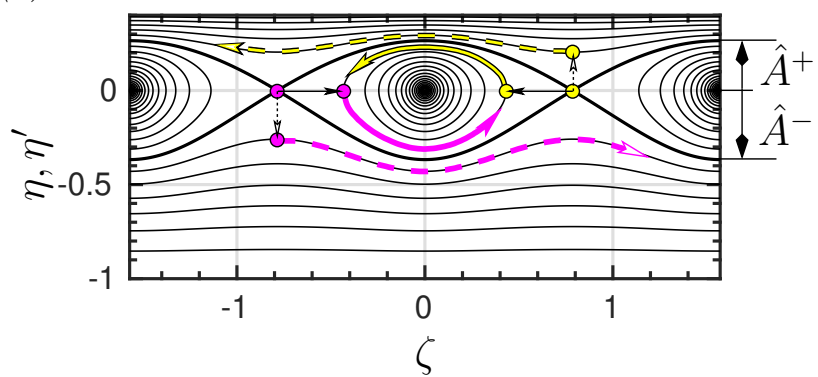

(c)

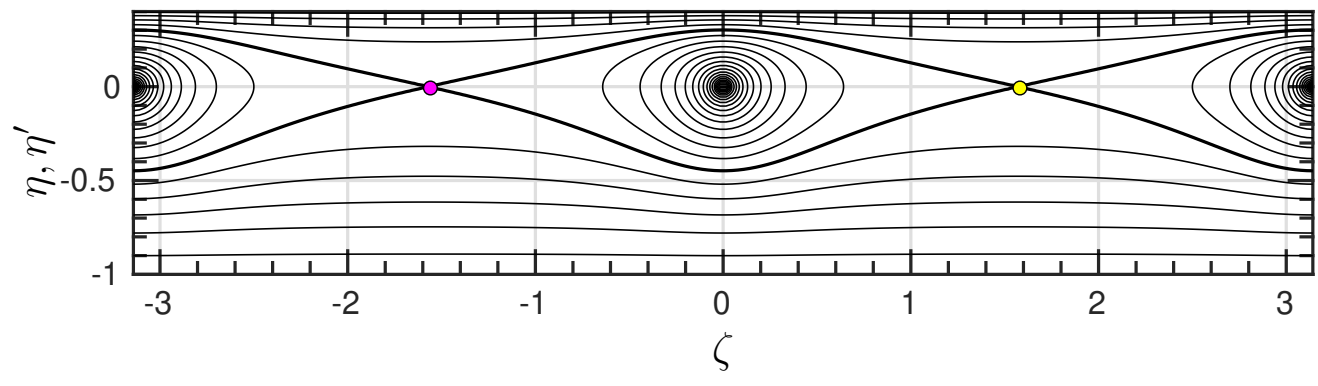

FIG. 6. Two periodic alleys of vortex rings: isolines $\hat{H}(\zeta, \eta)=$ cst for (a) $\hat{L}=0.1$, (b) $\hat{L}=0.5$ and (c) $\hat{L}=1$. The difference between two successive isocontours is $\Delta \hat{H}=0.2$. The locations of the two alleys are $(\zeta, \eta)$ and $\left(\zeta^{\prime}, \eta^{\prime}\right)=$ $\left(-\zeta, \sqrt{1-2 \eta-\eta^{2}}-1\right)$. The bottom axis $\eta=-1$ is the common symmetry axis of the rings. In the reference state, vortex rings have core size $\hat{a}_{0}=0.01$. Graph (b) shows typical leapfrogging vortex trajectories obtained when the initial perturbation is axial (along $\zeta$, solid lines), and overtaking trajectories when it is radial (along $\eta$, dashed lines); radial amplitudes $\hat{A}^{+}>0$ and $\hat{A}^{-}<0$ are also defined. Movies of typical leapfrogging and overtaking vortex motions (case $\left.L=0.5, a_{0}=0.06\right)$ presented simultaneously in $3 \mathrm{D}$ perspective and in the $(\zeta, \eta)$-plane are provided in the Supplemental Material [1].

and radius $R_{0}$ ) as:

$$
\begin{aligned}
\hat{H}\left(\eta, \eta^{\prime}, \zeta\right) \equiv H / \Gamma^{2}= & (1+\eta)\left[\frac{3}{4} \log (1+\eta)-\frac{1}{2} \log a_{0}+F\right] \\
& +\left(1+\eta^{\prime}\right)\left[\frac{3}{4} \log \left(1+\eta^{\prime}\right)-\frac{1}{2} \log a_{0}+F\right] \\
& +(1+\eta) I(\eta)+\left(1+\eta^{\prime}\right) I\left(\eta^{\prime}\right)+\sqrt{(1+\eta)\left(1+\eta^{\prime}\right)} J\left(\eta, \eta^{\prime}, \zeta\right),
\end{aligned}
$$

where the constant $F$ (defined in appendix A) depends on the internal vortex structure. The dimensionless functions $I$ and $J$ are defined by

$$
\begin{aligned}
I(\eta) & =\sum_{k=1}^{\infty}\left[\left(\frac{2}{\alpha_{k}}-\alpha_{k}\right) \mathscr{K}\left(\alpha_{k}\right)-\frac{2}{\alpha_{k}} \mathscr{E}\left(\alpha_{k}\right)\right], \quad \alpha_{k}^{2} \equiv \frac{(1+\eta)^{2}}{\pi^{2} \hat{L}^{2} k^{2}+(1+\eta)^{2}}, \\
J\left(\eta, \eta^{\prime}, \zeta\right) & =\sum_{k=-\infty}^{+\infty}\left[\left(\frac{2}{\beta_{k}}-\beta_{k}\right) \mathscr{K}\left(\beta_{k}\right)-\frac{2}{\beta_{k}} \mathscr{E}\left(\beta_{k}\right)\right], \quad \beta_{k}^{2} \equiv \frac{(1+\eta)\left(1+\eta^{\prime}\right)}{(\zeta+\pi \hat{L} k)^{2}+\left[1+\frac{1}{2}\left(\eta+\eta^{\prime}\right)\right]^{2}},
\end{aligned}
$$

where $\mathscr{K}(\alpha)$ and $\mathscr{E}(\alpha)$ are elliptical integrals of the first and second species (see appendix A for details). 
The conservation of the $z$-component of momentum $P_{z}$ imposes that perturbations $\eta$ and $\eta^{\prime}$ are of opposite signs and are linked by

$$
\eta^{\prime}=\sqrt{1-2 \eta-\eta^{2}}-1
$$

As already mentioned, this implies that the Hamiltonian $\hat{H}$ can again be written as a function of only two variables, namely $\eta$ and $\zeta$. Isolines of $\hat{H}(\eta, \zeta)$ are represented in figure 6; they provide the relative trajectories followed by coaxial rings.

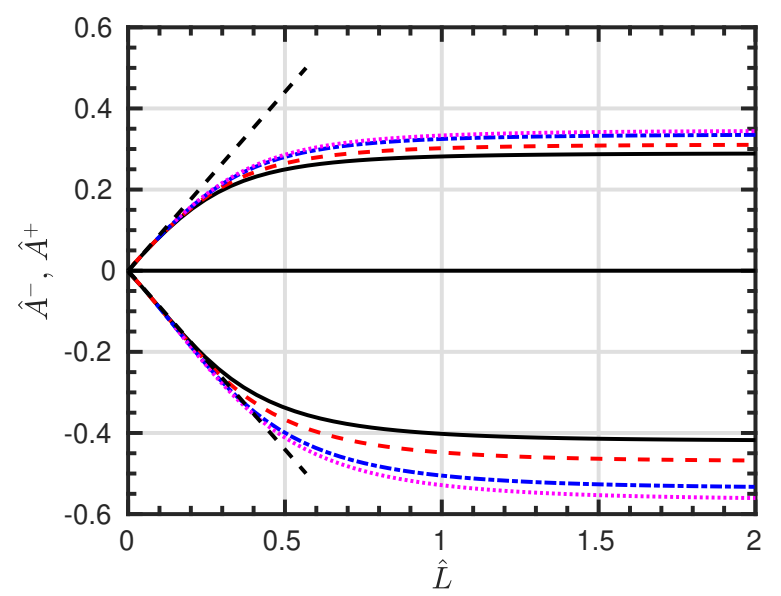

FIG. 7. Maximum amplitude of oscillations $\hat{A}^{-}$and $\hat{A}^{+}$for two vortex-ring alleys as a function of $\hat{L}$ for different core sizes $\hat{a}_{0}$. Values are $\hat{a}_{0}=0.001$ (black solid), 0.01 (red dashed), 0.06 (blue dot-dashed), 0.1 (magenta dotted). Black dashed straight lines: amplitudes $\hat{A}^{ \pm}= \pm 2 \pi s \hat{L}$ corresponding to two periodic alleys of two-dimensional point vortices.

As in section II, two types of trajectories are possible, reminiscent of leapfrog or overtaking (respectively represented with solid and dashed lines in figure 6b). When the radial displacement is small i.e. $|\eta| \ll 1$, relation (7) reduces to $\eta^{\prime}=-\eta$ : the symmetry between displacements of the two vortices prevailing in section II is recovered. For larger amplitudes, however, figure 6 indicates that this top-bottom symmetry (that is outward versus inward vortex) is nearly preserved for $\hat{L}=0.1$ (figure 6a), but clearly broken for larger values of $\hat{L}$ (figures $6 \mathrm{~b}$ and c). To be more quantitative, we define the maximal excursion $\hat{A}^{+}>0$ (resp. $\hat{A}^{-}<0$ ) of the outwards (resp. inwards) motion. For trajectories starting from the vicinity of the base state, i.e. of the hyperbolic points in figure 6 , vortices remain close to the separatrix, which then quantifies the oscillation amplitude (see definition in figure 6b). Amplitudes $\hat{A}^{ \pm}$are found to be monotonic functions of the helical pitch, as shown in figure 7. For the first model, the dimensional excursion was $s \ell$, with $s=\log (3+2 \sqrt{2}) /(4 \pi) \approx 0.14$; then $\hat{A}^{ \pm}= \pm 2 \pi s \hat{L}$ was unbounded and symmetric (dashed lines in figure 7 ). For the vortex ring alley, this situation is seen to hold for very small values of the pitch, typically $\hat{L} \leq 0.1$. For larger values, amplitudes $\hat{A}^{ \pm}$are asymmetric since equation (7) implies

$$
A^{ \pm}=\sqrt{1-2 A^{\mp}-A^{\mp^{2}}}-1,
$$

and they are found to both saturate with increasing $\hat{L}$. This implies that, during the dynamics, the ring radii always remain away from zero. The dependency of $\hat{A}^{ \pm}$with respect to the vortex core size $\hat{a}_{0}$ is also shown on figure 7: core size has a limited quantitative influence on these results. 
Taking curvature into account thus allows one to remain in an analytically tractable Hamiltonian framework and to describe some additional features such as the asymmetry observed in the oscillation amplitudes between the outward and the inward vortex. These amplitudes depend only slightly on the core size. However, situations at larger pitch values are clearly out of reach of the vortex ring approach and necessitate to introduce a model based on helical filaments.

\section{TWO HELICAL VORTICES AS A PAIR OF INVISCID HELICAL FILAMENTS}

The more advanced model includes torsion, curvature and core size and is based on a filamentary cut-off theory [22] in which helical symmetry is satisfied. This assumption requires that the core sizes $a_{p}$ be small compared to helix radii and pitch but the model is now valid for arbitrary values of the reduced pitch $\hat{L}$. In this framework, vortex filament $p$ is fully described by two coordinates: radius $r_{p}(t)$ and azimuthal angle $\varphi_{p}(t)$ (see equation 2). This model leads to somewhat involved computations which are detailed in appendix B, while the outline is given here. The motion imposed on vortex $p$ is the sum of the induction (B5) due to vortices $q \neq p$ and the self-induced velocity (B13). For two identical vortices of circulation $\Gamma$, this leads to two equations (B18)-(B19) for each vortex $p=1$ or 2 yielding the four-dimensional dynamical system

$$
\begin{aligned}
\frac{\mathrm{d} r_{1}}{\mathrm{~d} t} & =\mathscr{R}^{(1)}\left(r_{1}, r_{2}, \varphi_{1}, \varphi_{2}\right), \quad \frac{\mathrm{d} r_{2}}{\mathrm{~d} t}=\mathscr{R}^{(2)}\left(r_{1}, r_{2}, \varphi_{1}, \varphi_{2}\right), \\
\frac{\mathrm{d} \varphi_{1}}{\mathrm{~d} t} & =\mathscr{F}^{(1)}\left(r_{1}, r_{2}, \varphi_{1}, \varphi_{2}\right), \frac{\mathrm{d} \varphi_{2}}{\mathrm{~d} t}=\mathscr{F}^{(2)}\left(r_{1}, r_{2}, \varphi_{1}, \varphi_{2}\right),
\end{aligned}
$$

with

$$
\begin{aligned}
& \mathscr{R}^{(1)} \equiv \frac{\Gamma \gamma^{2}}{4 \pi} r_{2}\left[A_{1,2} \cos \left(\varphi_{1}-\varphi_{2}\right)-B_{1,2} \sin \left(\varphi_{1}-\varphi_{2}\right)\right] \\
& \mathscr{R}^{(2)} \equiv-\frac{\Gamma \gamma^{2}}{4 \pi} r_{1}\left[A_{1,2} \cos \left(\varphi_{1}-\varphi_{2}\right)-B_{1,2} \sin \left(\varphi_{1}-\varphi_{2}\right)\right], \\
\mathscr{F}^{(1)}= & \frac{\Gamma \gamma^{2}}{2 \pi}\left[\mathscr{G}_{1}\left(\gamma r_{1},|\gamma| \delta_{1} a_{1}\right)-\left(1+\gamma^{2} r_{1}^{2}\right) \mathscr{G}_{2}\left(\gamma r_{1},|\gamma| \delta_{1} a_{1}\right)\right] \\
& +\frac{\Gamma \gamma^{2}}{4 \pi}\left[F_{1,2}-\frac{r_{2}}{r_{1}} B_{1,2} \cos \left(\varphi_{2}-\varphi_{1}\right)+\frac{r_{2}}{r_{1}} A_{1,2} \sin \left(\varphi_{2}-\varphi_{1}\right)\right] \\
& +\frac{\Gamma \gamma^{4}}{4 \pi}\left[r_{1} r_{2} C_{1,2} \cos \left(\varphi_{2}-\varphi_{1}\right)-r_{1} r_{2} D_{1,2} \sin \left(\varphi_{2}-\varphi_{1}\right)-r_{2}^{2} F_{1,2}\right], \\
\mathscr{F}^{(2)}= & \frac{\Gamma \gamma^{2}}{2 \pi}\left[\mathscr{G}_{1}\left(\gamma r_{2},|\gamma| \delta_{2} a_{2}\right)-\left(1+\gamma^{2} r_{2}^{2}\right) \mathscr{G}_{2}\left(\gamma r_{2},|\gamma| \delta_{2} a_{2}\right)\right] \\
& +\frac{\Gamma \gamma^{2}}{4 \pi}\left[F_{2,1}-\frac{r_{1}}{r_{2}} B_{2,1} \cos \left(\varphi_{1}-\varphi_{2}\right)+\frac{r_{1}}{r_{2}} A_{2,1} \sin \left(\varphi_{1}-\varphi_{2}\right)\right] \\
& +\frac{\Gamma \gamma^{4}}{4 \pi}\left[r_{2} r_{1} C_{2,1} \cos \left(\varphi_{1}-\varphi_{2}\right)-r_{2} r_{1} D_{2,1} \sin \left(\varphi_{1}-\varphi_{2}\right)-r_{1}^{2} F_{2,1}\right],
\end{aligned}
$$


where $\gamma \equiv L^{-1}$ and the various coefficients are explicited in appendix B. Note that quantities $\mathscr{R}^{(1)}, \mathscr{R}^{(2)}$, $\mathscr{F}^{(1)}, \mathscr{F}^{(2)}$ depend only on $r_{1}, r_{2}$ and $\varphi_{1}-\varphi_{2}$ but not on $\varphi_{1}+\varphi_{2}$. Furthermore, the dynamical system (9) conserves $\Gamma\left(r_{1}^{2}+r_{2}^{2}\right)$ as for two alleys of vortex rings (see B7). Since $R_{0}$ is constant, variable $r_{2}$ is slaved to $r_{1}$ via $r_{2}^{2}=2 R_{0}^{2}-r_{1}^{2}$ : the problem is thus reduced to a two-dimensional dynamical system for variables $r_{1}$ and $\Delta \varphi_{1} \equiv \varphi_{1}-\varphi_{2}$ only:

$$
\frac{\mathrm{d} r_{1}}{\mathrm{~d} t}=\mathscr{R}^{(1)}, \quad \frac{\mathrm{d} \Delta \varphi_{1}}{\mathrm{~d} t}=\mathscr{F}^{(1)}-\mathscr{F}^{(2)}, \quad \text { with } \quad r_{2}=\sqrt{2 R_{0}^{2}-r_{1}^{2}} .
$$

The global rotation rate $\omega \equiv \mathrm{d}\left[\frac{1}{2}\left(\varphi_{1}+\varphi_{2}\right)\right] / \mathrm{d} t$ is unsteady but its value is slaved to $r_{1}$ and $\Delta \varphi_{1} \equiv \varphi_{1}-\varphi_{2}$. In the following we will not analyze this global rotation. Functions $\mathscr{F}^{(p)}$ depend on the core size $a_{p}$ through the arguments of functions $\mathscr{G}_{1}$ and $\mathscr{G}_{2}$ [see equations (11), (12) and (B12)]. The volume of the vortex core over a spatial period is equal to $\pi a_{p}^{2} C_{p}$ where $C_{p}(t)=|\ell|\left(1+\gamma^{2} r_{p}^{2}\right)^{1 / 2}$ denotes the length of the helical filament over this period. As the volume $\pi a_{p}^{2} C_{p}$ is conserved during vortex motion, the core size $a_{p}$ is in turn slaved to the helix radius $r_{p}$ :

$$
\frac{a_{p}^{2}(t)}{a_{p}^{2}(0)}=\frac{C_{p}(0)}{C_{p}(t)}=\sqrt{\frac{1+\gamma^{2} r_{p}^{2}(0)}{1+\gamma^{2} r_{p}^{2}(t)}} .
$$

Finally dimensionless factors $\delta_{1}$ or $\delta_{2}$ are linked to the internal velocity profiles in the corresponding vortex core via equation (B10): we always use the value for a Gaussian velocity profile $\delta_{1}=\delta_{2} \approx 0.8735$. When the vortex is stretched, these dimensionless parameters remain constant if one assumes self-similarity in the internal velocity profiles.

In the framework of the cut-off theory, the base state is given by two identical helical filaments of pitch $\ell$, axially distant of $\ell / 2$ (see figure 1 ) and steadily rotating at angular velocity $\omega_{\text {ref }}$ :

$$
\begin{gathered}
r_{1}=r_{2}=R_{0}, \quad \delta_{1}=\delta_{2}=\delta_{0}, \quad a_{1}=a_{2}=a_{0} \text { and } \varphi_{1}=\omega_{\mathrm{ref}} t, \varphi_{2}=\pi+\omega_{\mathrm{ref}} t \\
\omega_{\mathrm{ref}}=\frac{\Gamma \gamma^{2}}{2 \pi}\left[\mathscr{G}_{1}\left(\gamma R_{0},|\gamma| \delta_{0} a_{0}\right)-\left(1+\gamma^{2} R_{0}^{2}\right) \mathscr{G}_{2}\left(\gamma R_{0},|\gamma| \delta_{0} a_{0}\right)\right]+\frac{\Gamma}{2 \pi R_{0}^{2}}-\frac{\Gamma \gamma^{2}}{4 \pi}\left(1+\gamma^{2} R_{0}^{2}\right) G_{1,2} \\
G_{1,2} \equiv \int_{-\infty}^{+\infty} \frac{1+\cos \theta}{\left(2 \gamma^{2} R_{0}^{2}+2 \gamma^{2} R_{0}^{2} \cos \theta+\theta^{2}\right)^{3 / 2}} \mathrm{~d} \theta
\end{gathered}
$$

The above form solves the steady equation system because of the symmetries $B_{1,2}=B_{2,1}, C_{1,2}=C_{2,1}$, $F_{1,2}=F_{2,1}$ and because $A_{1,2}=A_{2,1}=0$ when $\varphi_{2}-\varphi_{1}=\pi$ (see B7). In order to study the stability of such a base state, it can be perturbed at initial time so that

$$
r_{1}=R_{0}(1+\eta), \quad r_{2}=R_{0}\left(1+\eta^{\prime}\right), \quad \varphi_{1}=\varepsilon, \quad \varphi_{2}=\pi-\varepsilon .
$$

Radial perturbations $\eta$ and $\eta^{\prime}$ in (18) are related through the last condition of (13), which again yields relation (7). For azimuthal displacements, only the difference $\Delta \varphi_{1}$ matters, so that a global shift makes it possible to use a single parameter $\varepsilon$. This corresponds to a relative displacement along the $z$-axis of $\Delta \hat{z}_{1}=\pi \hat{L}-2 \hat{L} \varepsilon$ where $\Delta \hat{z}_{1} \equiv \hat{z}_{1}-\hat{z}_{2}=-\hat{L} \Delta \varphi_{1}$. 
(a)

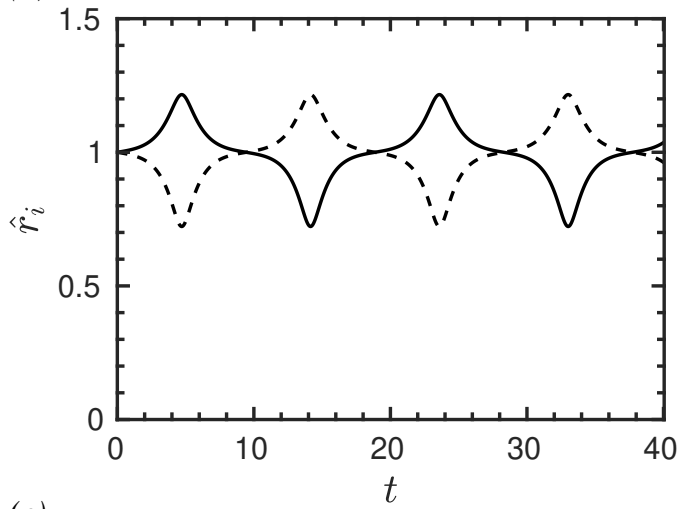

(c)

$t=0$

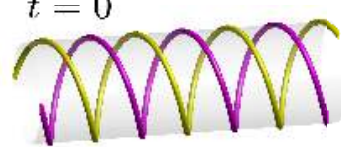

10

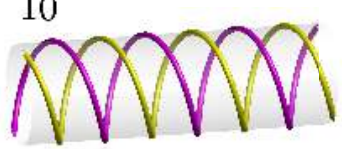

(d)

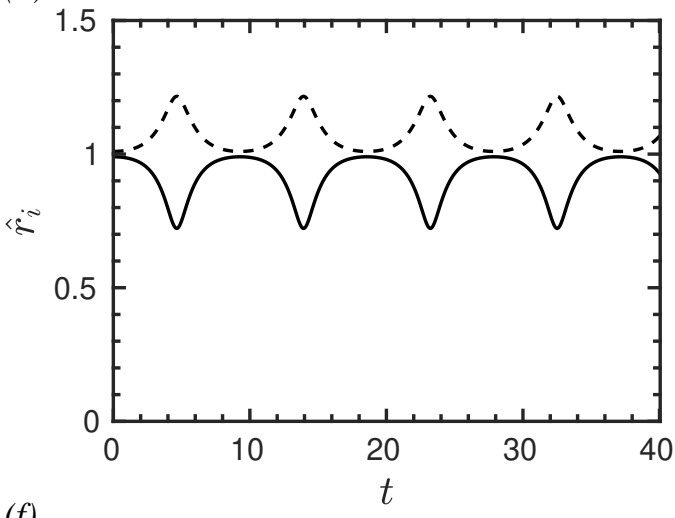

(f)

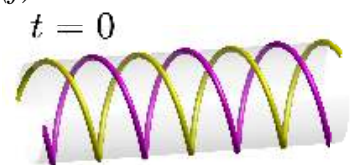

10

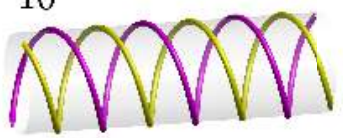

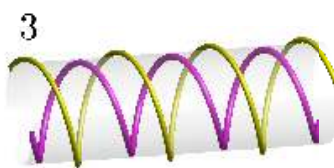

12

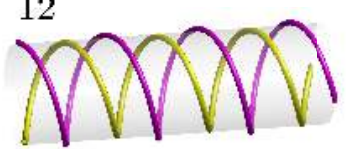

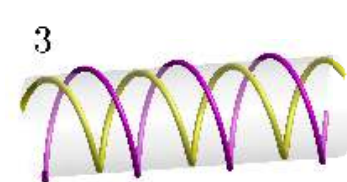

12

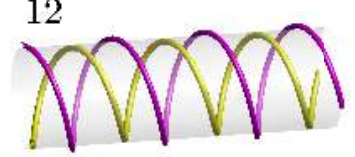

(b)

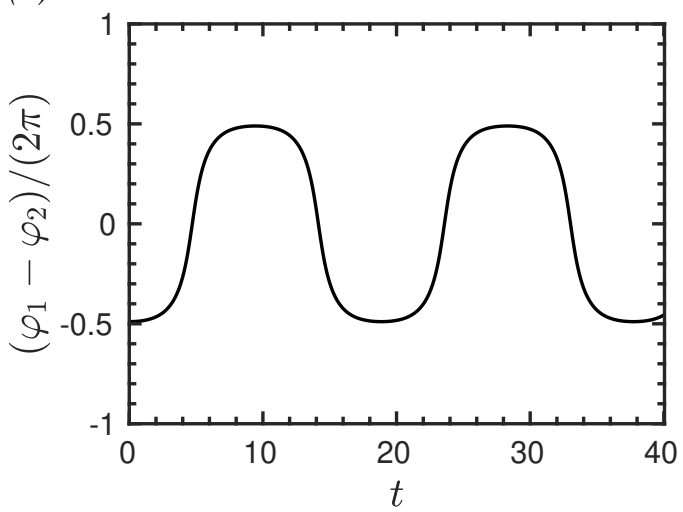

5

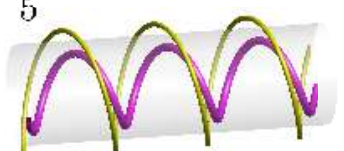

14
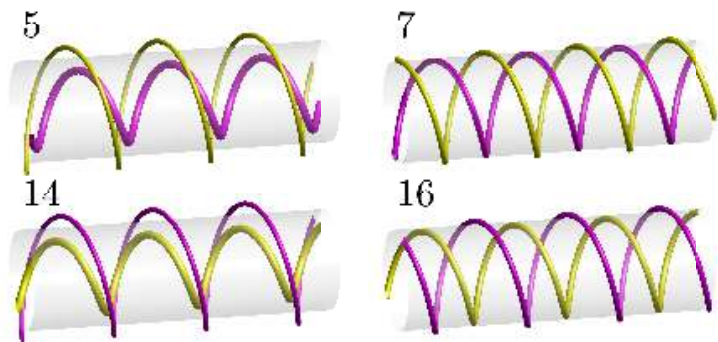

16

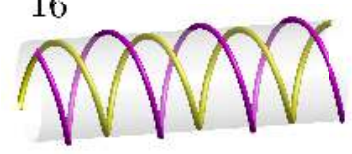

(e)
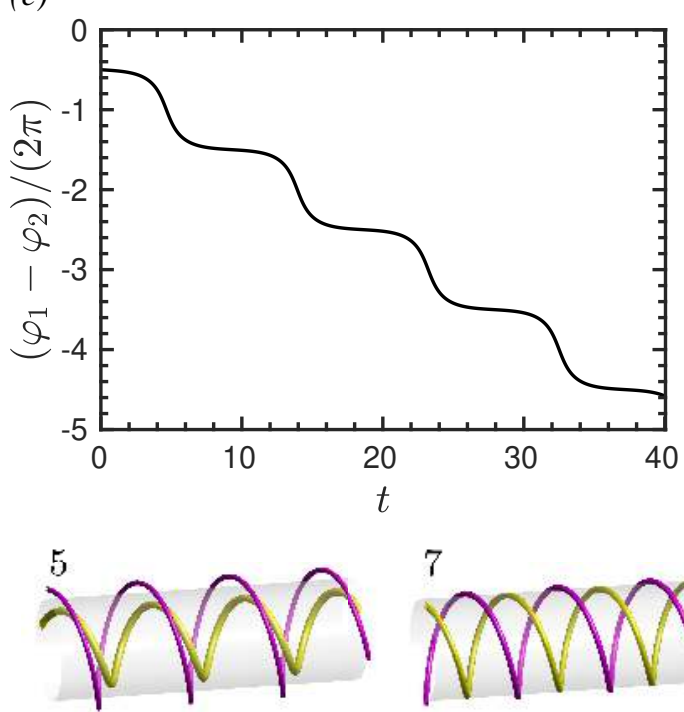

14
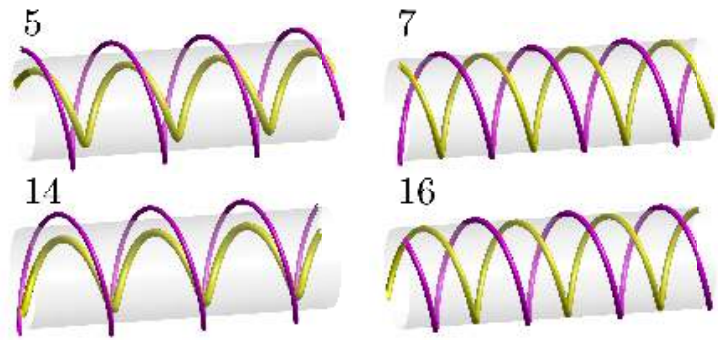

16

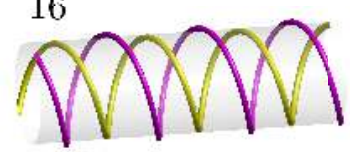

FIG. 8. Inviscid cut-off filament approximation. Two helical vortices of pitch $\hat{L}=0.3$, core size $a_{0}=0.06$ initially perturbed with (a)-(c) an axial perturbation $|\varepsilon|=0.01$ (d)-(f) a radial perturbation $|\eta|=0.01$. The helix radii are displayed in (a) and (d) and difference $\left(\varphi_{1}-\varphi_{2}\right) /(2 \pi)$ is plotted in (b) and (e). Snapshots of the 3D vortices at several times are represented in (c) and (f), while the corresponding movies are provided in the Supplemental Material [1]. 
A. Inviscid dynamics at low pitch $\hat{L} \lesssim 0.3$

(a)

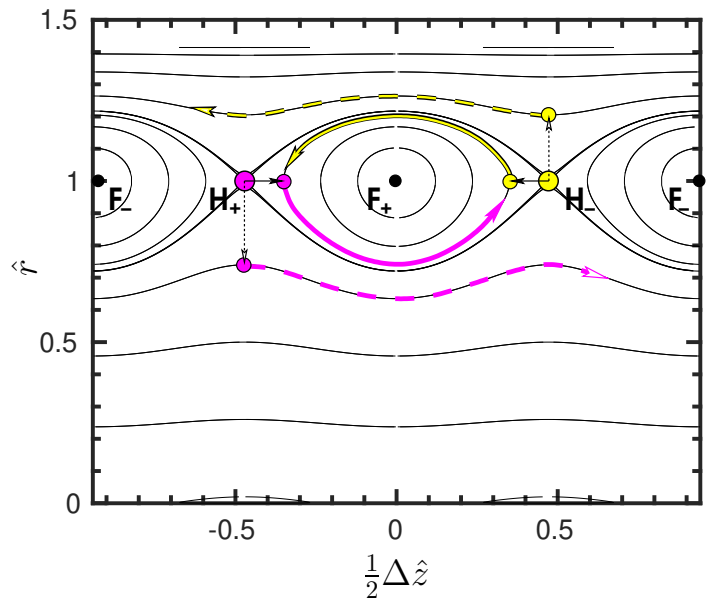

(b)

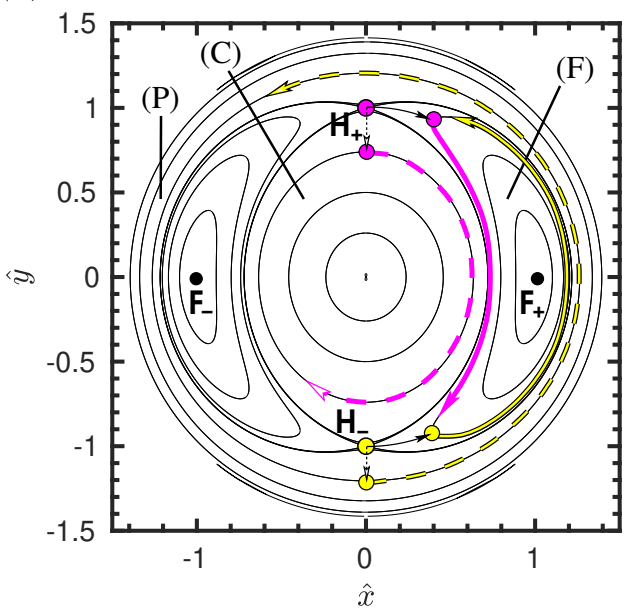

FIG. 9. Vortex trajectories for two helical filaments of reduced pitch $\hat{L}=0.3$ and core size $\hat{a}_{0}=0.06$ (a) when displayed in a meridional plane using Cartesian coordinates $\left(\frac{1}{2} \Delta \hat{z}, \hat{r}\right) ;(\mathrm{b})$ when displayed in a plane $(\hat{x}, \hat{y})$ orthogonal to the symmetry axis using polar coordinates $\left(\hat{r}, \frac{1}{2} \Delta \varphi\right)$. Hyperbolic points $\mathrm{H}_{ \pm}$correspond to the base state. Black dots denote elliptic points $\mathrm{F}_{ \pm}$. Typical leapfrogging vortex motion obtained when the initial perturbation is axial (solid lines), and overtaking motion when it is radial (dashed lines) are represented. Note that in this section, such diagrams are obtained by time integrations of vortex trajectories starting from a set of arbitrarily chosen initial conditions, rather than by drawing regularly spaced levels of a Hamiltonian function (hence levels are arbitrary).

The cut-off filament approximation predicts that at low pitch values $\hat{L} \lesssim 0.3$ the dynamics of two helical filaments is very similar to the dynamics of point-vortex or vortex-ring alleys: when vortices are slightly displaced axially $(\eta=0, \varepsilon \ll 1)$, leapfrog dynamics are observed (figures $8 \mathrm{a}-\mathrm{c}$ ). When they are slightly displaced radially ( $\varepsilon=0$ and $\eta \ll 1$ ), overtaking occurs instead (figures $8 \mathrm{~d}-\mathrm{f}$ ). To account for this property, one may characterize the orbits of the two helical vortex filaments in a phase space representation that yields patterns similar to those obtained for point-vortex and vortex-ring alley models: a Cartesian representation in a meridional plane introduced in figure 9a, for which vortices 1 and 2 have respectively coordinates $\left(\frac{1}{2} \Delta \hat{z}_{1}, \hat{r}_{1}\right)$ and $\left(\frac{1}{2} \Delta \hat{z}_{2}, \hat{r}_{2}\right)$, with $\Delta \hat{z}_{2} \equiv \hat{z}_{2}-\hat{z}_{1}=-\Delta \hat{z}_{1}$. This representation is related to a cut in a meridional plane where the origin of the $z$-axis is placed at any time at midpoint between the two vortex axial positions. This time-dependent representation cancels out the global rotation and is adapted when $\hat{L}$ is small or when vortices are located away from the symmetry axis $\hat{r}=0$. The discussion for the orbits starting near the saddle points called here $\mathrm{H}_{ \pm}$is similar to that of sections II and III, leading to the same conclusions about overtaking or leapfrogging dynamics at low reduced pitch.

On the quantitative side, for the reduced pitch value $\hat{L}=0.3$, the amplitude of outward (resp. inward) motion is given by the maximum (resp. minimum) value of $\hat{r}_{i}(t)-1$, namely $\hat{A}^{+}=0.22$ (resp. $A^{-}=-0.28$ ). The vortex ring alley approximation for the same core size $a_{0}=0.06$ gives the value 0.21 (resp. -0.27 ) while the point vortex alley approximation yields 0.26 (resp. -0.26). The point-vortex model shown previously to be pertinent for helical vortices with $\hat{L} \lesssim 0.1$ is no more adequate for $\hat{L}=0.3$. The vortex-ring alley model, 
which has the advantage of being analytically tractable, is yet pertinent to quantitatively describe helical vortices up to $\hat{L} \lesssim 0.3$, but proves to be no more valid at larger pitches, as shown below.

\section{B. Inviscid dynamics at larger pitches $\hat{L} \gtrsim 0.3$}

The Cartesian representation of figure 9a becomes inadequate at larger pitch as the dynamics near the centerline becomes more important. We thus introduce an alternative representation based on polar coordinates (see figure 9b): the two filaments are cut in a plane perpendicular to the symmetry axis, the whole system being rotated of an angle $\frac{1}{2}\left(\varphi_{1}+\varphi_{2}\right)$ which cancels out the global rotation. When the location $\left(\hat{r}_{1}, \frac{1}{2} \Delta \varphi_{1}\right)$ of vortex 1 is known, the location $\left(\hat{r}_{2}, \frac{1}{2} \Delta \varphi_{2}\right)$ of vortex 2 is obtained as follows: $\hat{r}_{2}$ results from $\hat{r}_{1}^{2}+\hat{r}_{2}^{2}=2$, and the angle of vortex 2 is obtained by mirror symmetry with respect to the horizontal axis since $\Delta \varphi_{2}=-\Delta \varphi_{1}$. Note that if point $\left(\hat{r}_{1}, \frac{1}{2} \Delta \varphi_{1}\right)$ is occupied, the point $\left(\hat{r}_{1}, \frac{1}{2} \Delta \varphi_{1}+\pi\right)$ is also a possible state which is fully equivalent. For small $\hat{L}$, (figure $9 \mathrm{~b}$ ), the phase space in this new representation is structured by two saddle points $\mathrm{H}_{ \pm}$at $(\hat{x}, \hat{y})=(0, \pm 1)$ representing the unstable base state, as well as two elliptic points $\mathrm{F}_{ \pm}$at $( \pm 1,0)$. The regions denoted by $(\mathrm{F})$ attached to these elliptic points contain the leapfrogging cycles (solid lines in figure 9b). Overtaking cycles (dashed lines) occur when one vortex travels in the center region (C) while its companion travels around in the peripheral region $(\mathrm{P})$.

As $\hat{L}$ increases (see $\hat{L}=0.7$ in figure 10a), two elliptic points $\mathrm{E}_{ \pm}$located at $(0, \pm e)$ get separated from the origin, that is distance $|e|$ increases with $\hat{L}$. In addition, each region (E) surrounding each point $\mathrm{E}_{ \pm}$grows in size in region $(\mathrm{C})$. Inside the peripheral region $(\mathrm{P})$, there exist two conjugate regions $\left(\mathrm{E}^{\prime}\right)$ pertaining to two elliptic points $\mathrm{E}_{ \pm}^{\prime}$ located at $\left(0, \pm e^{\prime}\right)$. As $\hat{L}$ increases, these regions grow such that $e^{2}+e^{\prime 2}=2$, hence $e^{\prime}$ decreases from $\sqrt{2} \approx 1.41$ while $e$ increases from 0 . So far these new islands are situated far from the saddle points $\mathrm{H}_{ \pm}$and from the associated separatrix, so that they do not much influence the dynamics of vortices when slightly perturbed from the base state. This justifies that, compared to the dynamics at $\hat{L}=0.3$, the dynamics obtained at $\hat{L}=0.7$ are not that qualitatively different: the time evolutions plotted in figure 11 again show leapfrogging (resp. overtaking) depending on the initial axial (resp. radial) shift of the vortices. On the quantative side however, the amplitude of motion at $\hat{L}=0.7$ is much larger than at $\hat{L}=0.3$.

However, as $\hat{L}$ further increases, the new island (E) in figure 10 grows at the expense of the complementary region inside $(\mathrm{C})$, which becomes thinner and thinner so as to disappear at a specific value $\hat{L}_{0}$. For the core size $\hat{a}_{0}=0.06$, this value is $\hat{L}_{0} \approx 0.85$ (case of figure $10 \mathrm{~b}$ ). The same occurs inside the peripheral region (P). At $\hat{L}=\hat{L}_{0}$, the saddle points $\mathrm{H}_{ \pm}$are directly connected to the origin: slight perturbations of the base state lead the inner vortex to go towards the center of the domain where the helix radius vanishes. Such behaviour is illustrated in figure 12.

For $\hat{L}>\hat{L}_{0},|e|$ keeps increasing with $\hat{L}$, the elliptic points $\mathrm{E}_{ \pm}$and $\mathrm{E}_{ \pm}^{\prime}$ continue to migrate towards the saddle points $\mathrm{H}_{ \pm}$while the size of the regions associated to these elliptic points now decreases. Figure 10c illustrates that, at $\hat{L}=1$, trajectories coming from the vicinity of the saddles now remain trapped in closed regions situated in separated (upper and lower) half-planes. This gives rise to a new regime that we call the fluttering regime. Small axial and radial perturbations cannot now induce any exchange cycle, that is to say no leapfrogging nor overtaking but still induce different dynamics. Axial perturbations induce fluttering with radial inward/outward alternation (solid lines in figure 10c). By contrast, radial perturbations induce fluttering without alternation (dashed lines). This can be followed over time in figure 13. While radial motions at $\hat{L}=1$ are almost identical as at $\hat{L}=0.7$, azimuthal motions are completely different: time variations of $\varphi_{1}-\varphi_{2}$ (or equivalently of $\hat{z}_{1}-\hat{z}_{2}$ ) are relatively abrupt, but of very limited amplitudes, so that 
(a)

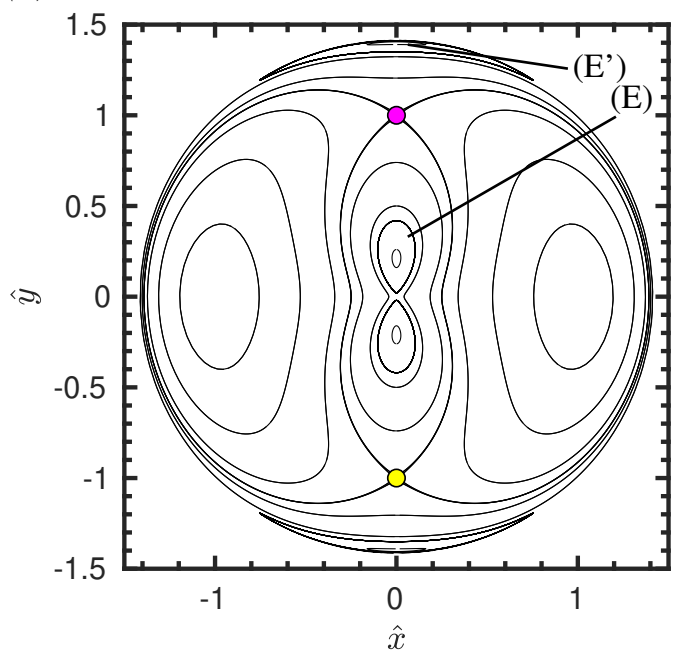

(c)

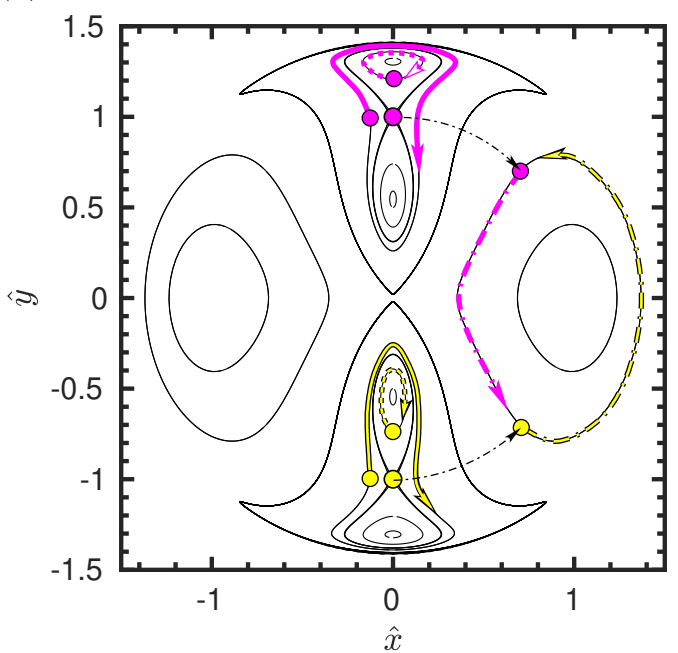

(b)

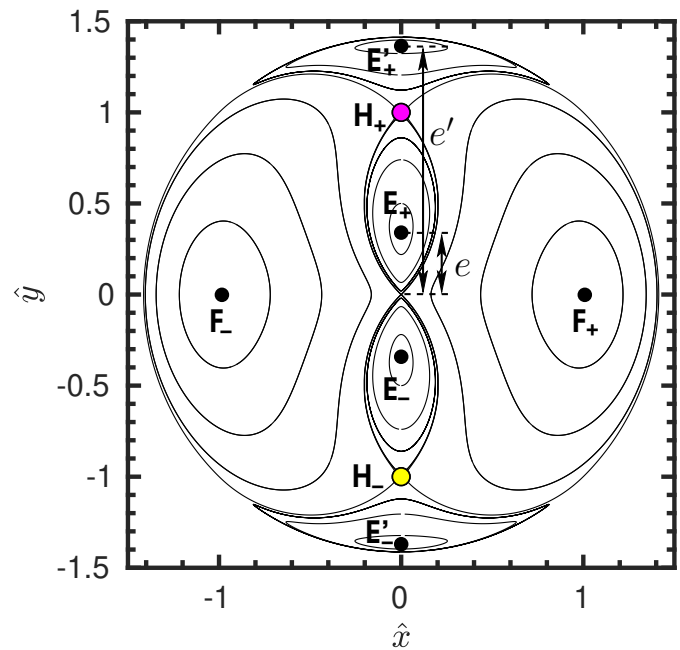

(d)

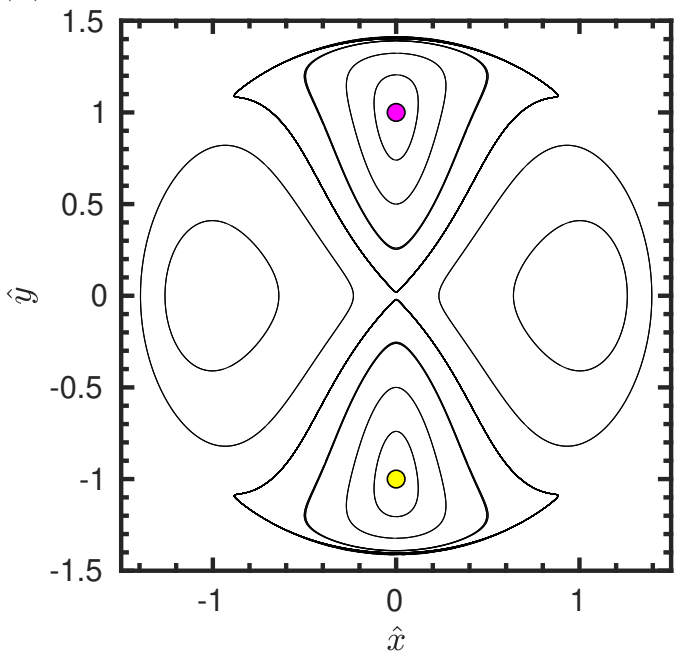

FIG. 10. Polar representation for vortices with equal core size $\hat{a}_{0}=0.06$ and reduced pitch (a) $\hat{L}=0.7$, (b) $\hat{L}=0.85$, (c) $\hat{L}=1$ and (d) $\hat{L}=1.3$. Graph $\mathrm{b}$ shows the elliptic points $\mathrm{E}_{ \pm}, \mathrm{E}_{ \pm}^{\prime}$ and $\mathrm{F}_{ \pm}$, as well as the saddle points $\mathrm{H}_{ \pm}$. Graph $\mathrm{c}$ shows typical fluttering motion with inward/outward radial alternation obtained when the initial perturbation is axial (solid color lines), and fluttering without radial alternation when it is radial (dashed lines). Leapfrogging motion (dot-dashed color lines) can also be obtained for large amplitude initial shifts from the base state.

the vortices remain on opposite "sides" with respect to the symmetry axis (figures $13 \mathrm{~b}$ and e). For $\hat{L}>\hat{L}_{0}$, the fluttering dynamics is thus mainly characterized by periodic radial excursions of the vortices, with or without radial inward/outward alternation, but no change in their interwaving order occurs.

Yet, it is noteworthy that large amplitude initial perturbations in the axial direction allow the two vortices to 
(a)

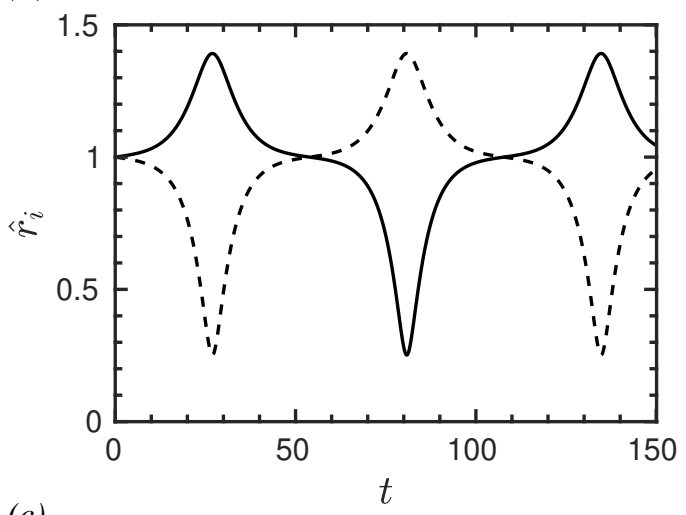

(c)

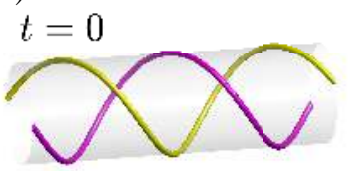

(d)

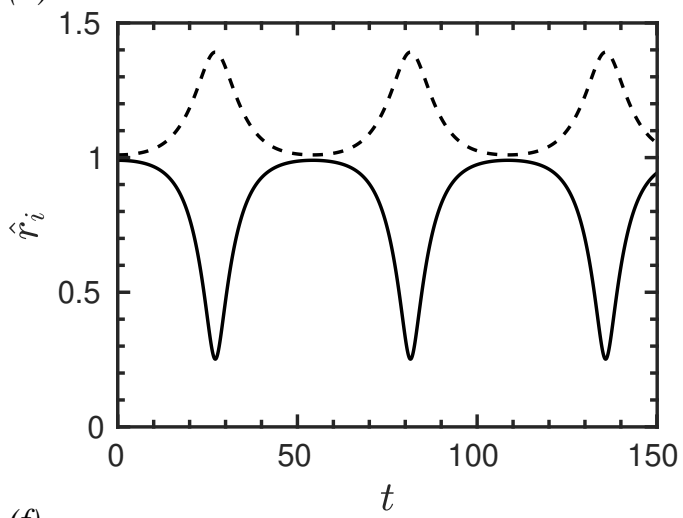

(f)

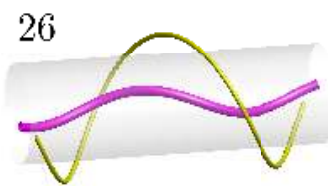

(e)

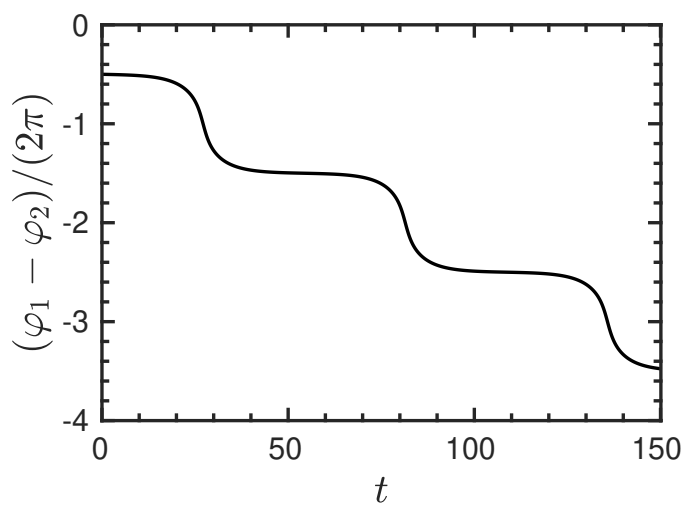

52

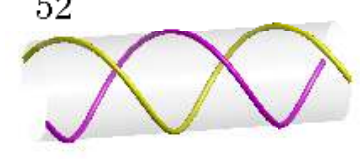

(b)

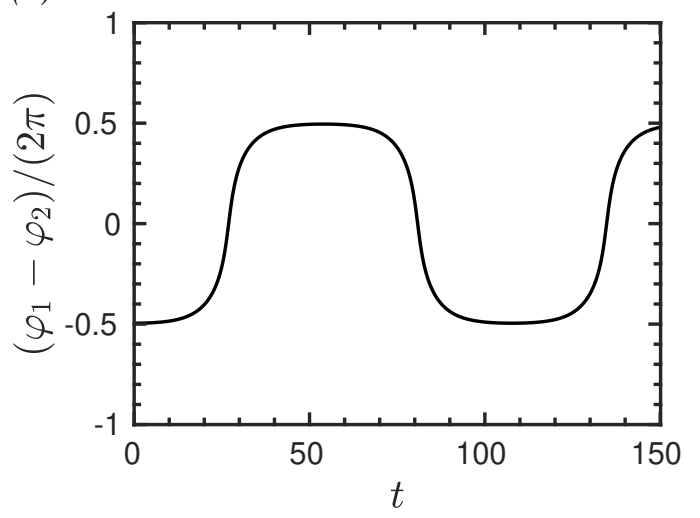

52
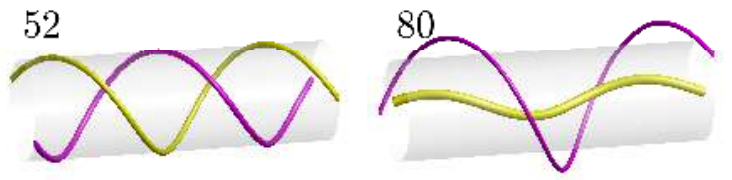

80

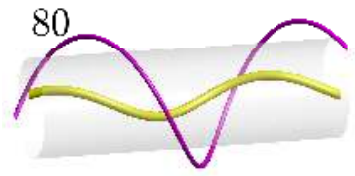

FIG. 11. Same as figure 8 but for helical pitch $\hat{L}=0.7$.

reach one of the regions attached to the elliptic points $\mathrm{F}_{ \pm}$(dot-dashed lines in figure 10c). In such a case, leapfrog dynamics can still occur, as shown by the time evolution presented in figure 14. The dynamics are then more rapid and regular, both vortices being close one to the other and the trajectories remaining far from hyperbolic points. Such situation is encountered in the DNS simulations [11, 25], e.g. near merging; when merging is over, vortices have reached the elliptic points $\mathrm{F}_{ \pm}$. On the contrary, overtaking dynamics 
(a)

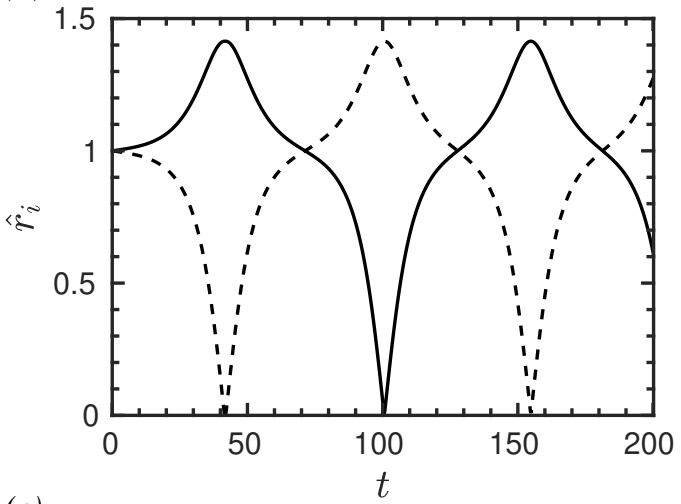

(c)

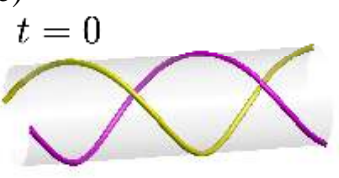

35

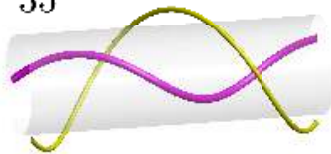

(b)
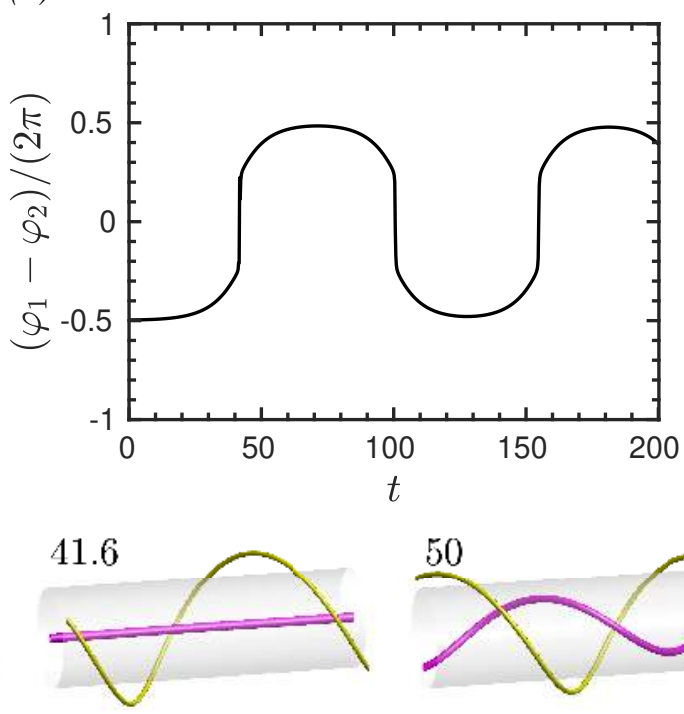

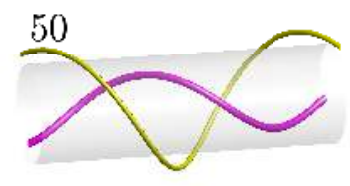

50

FIG. 12. Same as figure 8 but for helical pitch $\hat{L}=0.85$ (only the axial perturbation case).

cannot occur when $\hat{L}>\hat{L}_{0}$ since the peripheral region (P) has now disappeared.

As $\hat{L}$ is further increased, the elliptic points $\mathrm{E}_{ \pm}$and $\mathrm{E}_{ \pm}^{\prime}$ collide with the saddle points $\mathrm{H}_{ \pm}$at a critical value $\hat{L}=\hat{L}_{\mathrm{c}}$. For $\hat{L}>\hat{L}_{\mathrm{c}}$, there are 4 elliptic points left: two points at $(\hat{x}, \hat{y})=(0, \pm 1)$ yielding neutrally stable oscillations around the base state, and the two points $F_{ \pm}$at $( \pm 1,0)$ still allowing for leapfrog far from the equilibrium state (see diagram at $\hat{L}=1.3$ in figure 10d).

The transition from leapfrog/overtaking behavior at small $\hat{L}=0.3$ or 0.7 to fluttering at $\hat{L}=1$ may also be followed when looking at vortex trajectories in a plane perpendicular to the symmetry axis, thereby restoring the global rotation absent in the previous representation. In figure 15, only a part of the first cycle in shown, for the case where the base state has been perturbed in the axial direction. The increase of radial excursion is clearly seen when comparing $\hat{L}=0.3$ and $\hat{L}=0.7$ (respectively figure $15 \mathrm{a}$ and $\mathrm{b}$ ). As $\hat{L}$ increases, the inward vortex gets closer and closer to the symmetry axis as it loops around it during the exchange cycle: the size of the loop gradually reduces in that plane. At the specific pitch value $\hat{L}_{0}$ (figure $15 \mathrm{c}$ ), the trajectory along the inner loop goes through the symmetry axis. When $\hat{L}$ is further increased, the loop keeps reducing and may unfold (see figure $15 \mathrm{~d}$ at $\hat{L}=1$ ). The inner excursion of the vortex is no more associated to a loop around the axis: in the fluttering regime, both vortices remain in two distinct half-planes while oscillating radially. 

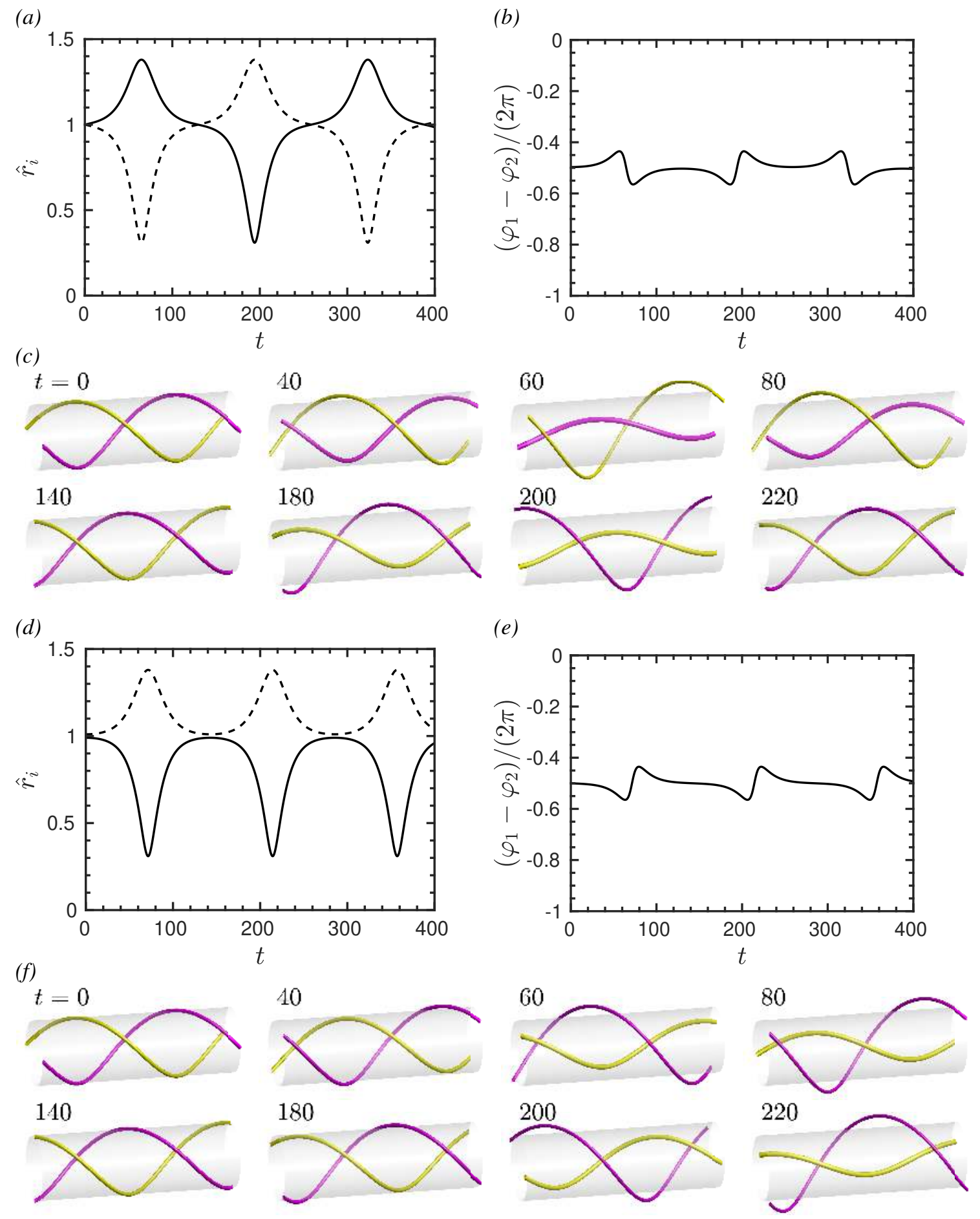

FIG. 13. Same as figure 8 but for helical pitch $\hat{L}=1$. 
(a)

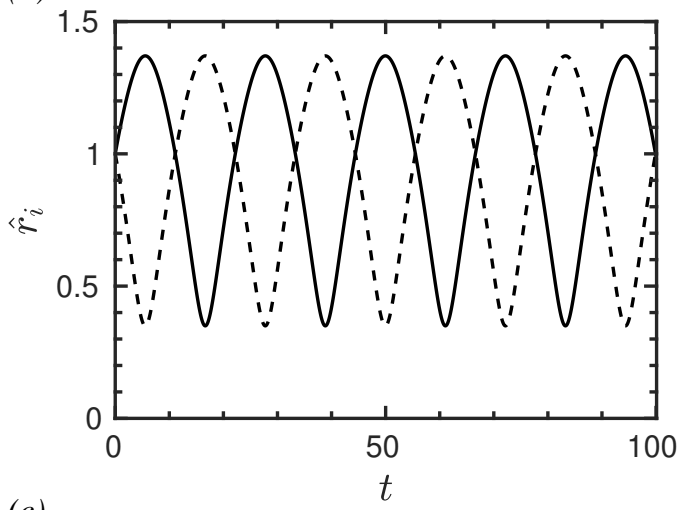

(c)

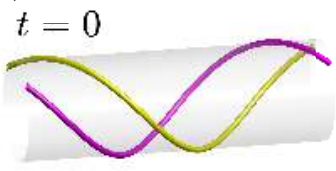

12

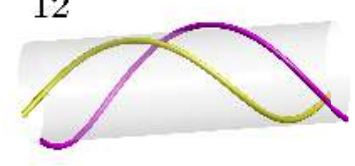

3

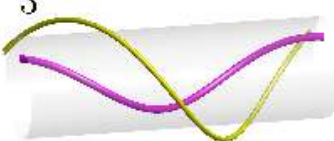

15

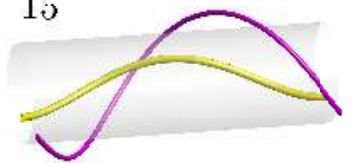

(b)

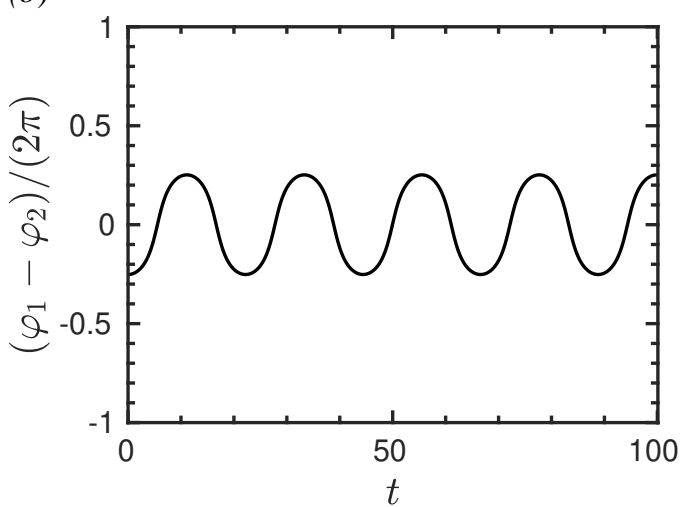

6

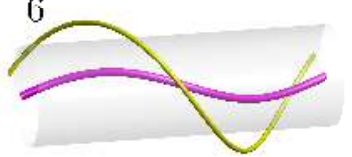

18

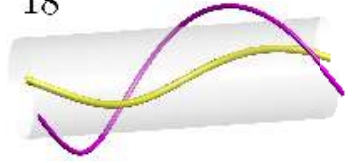

9

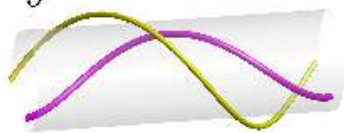

21

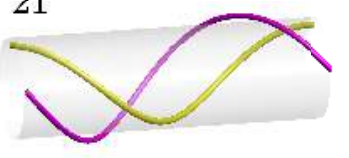

FIG. 14. Leapfrog triggered by an initial axial vortex shift of large amplitude $|\varepsilon|=\pi / 4$. (a) Helix radii, (b) azimuthal difference $\left(\varphi_{1}-\varphi_{2}\right) /(2 \pi)$ and (c) time sequence, for two helical vortices of pitch $\hat{L}=1$, core size $a_{0}=0.06$ in the inviscid cut-off filament approximation. The movie correponding to (c) is provided in the Supplemental Material [1].

\section{Radial amplitudes of vortex motion}

Finally let us examine the dependence of amplitudes $\hat{A}^{+}$and $\hat{A}^{-}$with respect to pitch $\hat{L}$ and core size $\hat{a}_{0}$, as displayed in figure 16a. As already stated, these amplitudes are deduced from the maximal amplitude excursions obtained when integrating system (13) in time in the vicinity of the base state. Their meaning is also recalled in figure 15 .

Starting from small values of $\hat{L}$, amplitudes $\left|A^{ \pm}\right|$grow, essentially following the curves for point-vortex alleys as $\hat{L} \lesssim 0.1$, for vortex-ring alleys as $\hat{L} \lesssim 0.3$, and then significantly deviating from them. It is seen on equation (8) that the maximum amplitude $\hat{A}^{+}$is bounded by $\hat{A}_{\max }^{+}=\sqrt{2}-1 \approx 0.41$, corresponding to $\hat{A}_{\min }^{-}=-1$. Contrary to the vortex ring alleys for which it was never observed (see figure 7), such extreme excursion can be achieved for two helical vortices and precisely takes place at the value $\hat{L}=\hat{L}_{0}$ (see figures 12 and $15 \mathrm{c})$. In figure 16 , curves $\hat{A}^{ \pm}(\hat{L})$ are plotted for several values of the core size $\hat{a}_{0}$ : their overall shape does not change much with $\hat{a}_{0}$. For $\hat{a}_{0}=0.06$, extreme excursion occurs at $\hat{L}_{0} \approx 0.85$ (at $\hat{L}_{0} \approx 1$ for core sizes smaller than 0.01). For $\hat{L}>\hat{L}_{0}$, amplitudes $\left|\hat{A}^{ \pm}\right|$decrease rapidly, until the critical pitch $\hat{L}_{\mathrm{c}}$ is reached, above which there is no more instability. For $\hat{a}_{0}=0.06$, stabilization occurs at $\hat{L}_{\mathrm{c}} \approx 1.15$ (at $\hat{L}_{\mathrm{c}} \approx 1.30$ for core sizes smaller than 0.01) $[11,19]$. 
(a)

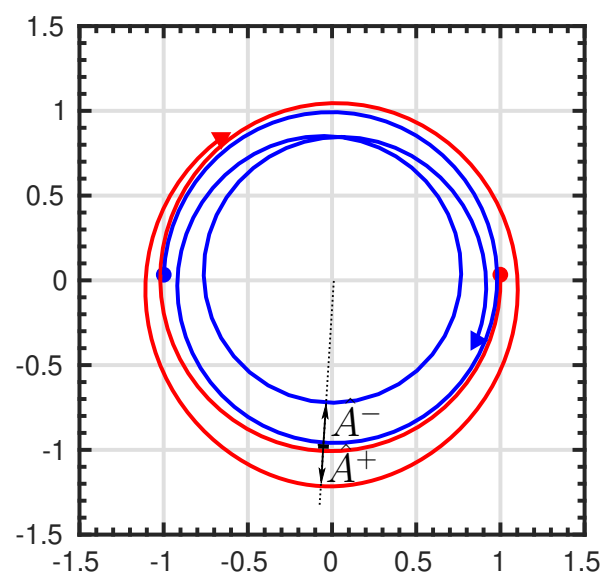

(c)

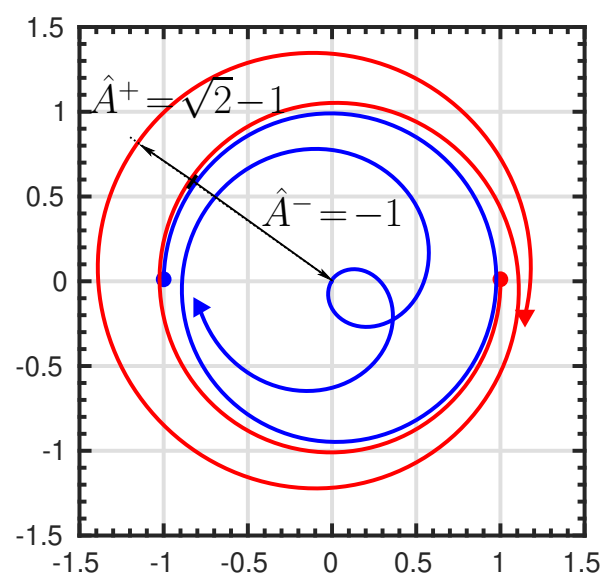

(b)

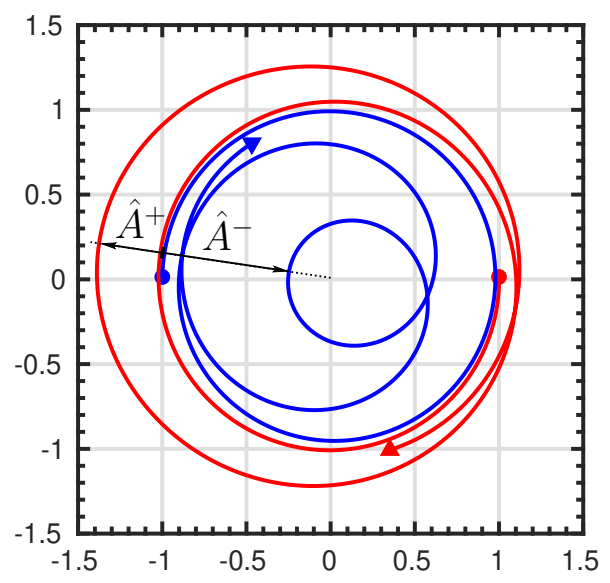

(d)

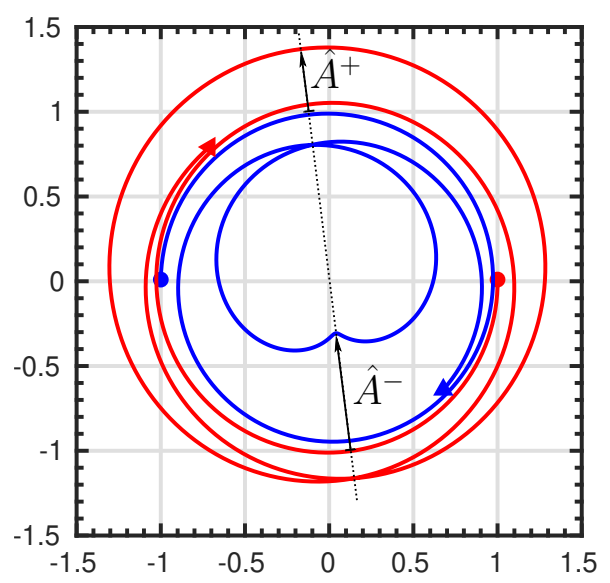

FIG. 15. Trajectories of the two vortices in a $(\hat{x}, \hat{y})$-plane orthogonal to the helix axis for pitch (a) $\hat{L}=0.3$, (b) $\hat{L}=0.7$, (c) $\hat{L}=\hat{L}_{0}=0.85$, (d) $\hat{L}=1$. The plots correspond to most of the initial cycle of the simulations presented in figures 8 , 11,12 and 13, graphs a and $b$ (axial initial perturbation). The two dots denote the initial vortex locations. The black arrows show the position of the vortices at maximal excursion and the corresponding radial amplitudes $\hat{A}^{ \pm}$from the base state (i.e. from the circle of unit radius).

These new results may be confronted to numerical and experimental results. First, comparisons with DNS results in the helical symmetry framework are displayed in figure 16b for different helical pitches $\hat{L}$. DNS are performed at $R e=\Gamma / v=5000$ ( $v$ denotes the kinematic viscosity of the fluid) with initial core sizes $\hat{a}(t=0)=0.06$, and small radial shift from the base state. At small pitches where the dynamics are rapid, the agreement between DNS and the inviscid cut-off theory at $\hat{a}_{0}=0.06$ is perfect. Yet as the pitch is increased above 0.6, DNS results depart from this theoretical curve. For increasing pitch, the dynamics become progressively slower and the core size in the DNS significantly increases during the very first cycle of evolution. DNS amplitudes then better agree with theoretical curves relative to larger core sizes, for 
(a)

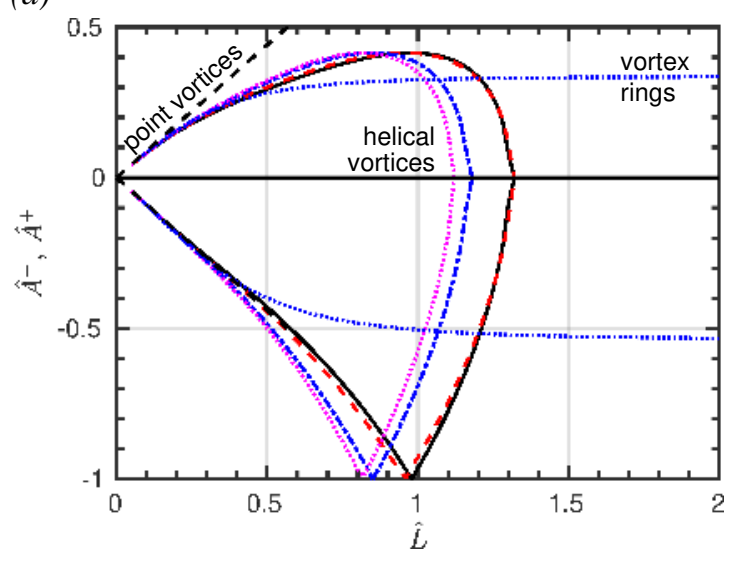

(b)

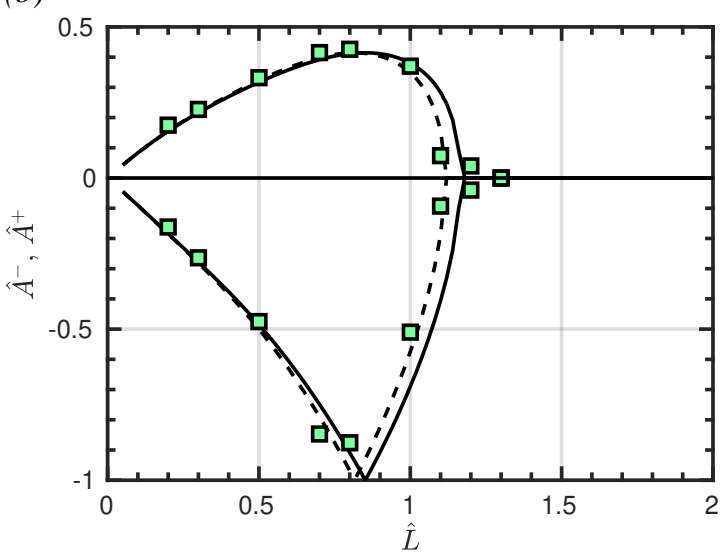

FIG. 16. Dynamics of two helical vortices: maximum radial amplitudes of oscillations $\hat{A}^{-}$and $\hat{A}^{+}$for a small perturbation of the base state, as a function of $\hat{L}$. (a) Inviscid cut-off simulations with core size $\hat{a}_{0}=0.001$ (black solid), 0.01 (red dashed), 0.06 (blue dot-dashed), 0.1 (magenta dotted); black dashed straight lines: two periodic alleys of point vortices; blue dotted lines: two vortex ring alleys with core size $\hat{a}_{0}=0.06$. (b) Square symbols: radial amplitudes of the first cycle in the DNS at $R e=5000$ for $\hat{a}(t=0)=0.06$; lines: cut-off for $\hat{a}_{0}=0.06$ (solid) and $\hat{a}_{0}=0.1$ (dashed).

instance $\hat{a}_{0}=0.1$. Second, comparisons with experimental results in spatially developing two-blade rotor wakes have been performed by Quaranta et al. [21]. When transposed to the temporal framework and properly rescaled, the radial evolution of the vortices showed a fair agreement with the numerical simulation data (see their figure 26).

\section{MIMICKING VISCOUS EFFECTS}

Inviscid results may differ from DNS results for several reasons: viscosity causes core size and helix radius to evolve in time, and vortex merging involves a modification of vortex shape and circulation, effects which are not taken into account in the above models. It is observed in Navier-Stokes simulations that the overtaking dynamics, when present, is generally followed by leapfrog and eventually merging [25]. It is possible to heuristically emulate this transition between overtaking and leapfrog in the point-vortex model. When the merging process starts, the two vortices are getting closer, a process that occurs together with filamentation. Merging may be modeled as an energy transfer from self-energy to interaction energy. In the point vortex model, the Hamiltonian $\hat{H}$ does not represent the total kinetic energy but only the part of it linked to the interaction between vortices, the self-interactions being basically removed (for a single vortex, the Hamiltonian thus vanishes). The energy transfer is implemented onto dynamical equations (4) by adding an ad-hoc 
extra term corresponding to a gradient of $\hat{H}$ :

$$
\frac{\mathrm{d}}{\mathrm{d} t}\left(\begin{array}{l}
\xi \\
\eta
\end{array}\right)=\left(\begin{array}{c}
\frac{\partial \hat{H}}{\partial \eta} \\
-\frac{\partial \hat{H}}{\partial \xi}
\end{array}\right)+v^{\prime}\left(\begin{array}{l}
\frac{\partial \hat{H}}{\partial \xi} \\
\frac{\partial \hat{H}}{\partial \eta}
\end{array}\right)
$$

where $0<v^{\prime} \ll 1$. This additional term causes $\hat{H}$ to increase since

$$
\frac{\mathrm{d}}{\mathrm{d} t} \hat{H}=v^{\prime}\left[\left(\frac{\partial \hat{H}}{\partial \xi}\right)^{2}+\left(\frac{\partial \hat{H}}{\partial \eta}\right)^{2}\right]>0 .
$$

When vortices are initially shifted in the transverse direction (along $\eta$ ), a clear transition between overtaking cycles and leapfrogging cycles is obtained (see figures $17 \mathrm{a}$ and $\mathrm{b}$ ). This transition is better seen in the $(\xi, \eta)$ plane: the trajectories, initiated outside the separatrix (blue and red circle in figure 17c), perform some cycles outside the separatrix before reaching an energy level which is associated with a trajectory inside the heteroclinic separatrix and thereafter remain in that region.

Viscosity effects in the DNS of two helical vortices are well mimicked by the above heuristic model, at least on a qualitative level. Morerover, there is a time signature for the transition between overtaking and leapfrogging: when approaching this transition, the trajectories generally get closer to the hyperbolic points such as $(\xi, \eta)=(0,0)$ and $(1,0)$. The system hence stays longer in that region, a feature clearly observed in the DNS [25]. The energy drift due to viscosity is thus responsible for the changing period of cycles especially when beginning or passing near the equilibrium state. It is also responsible for the sequencing of cycles: overtaking, then leapfrogging. Transition from leapfrogging to overtaking is observed neither in the simplified model nor in the DNS.

\section{CONCLUSION}

The present dynamical system approach explains the existence of leapfrog dynamics for two helical vortices in a simple way, but also unveils unveiled new dynamical features that we called overtaking and fluttering. At low pitch, typically $\hat{L} \lesssim 0.3$, radial perturbations favour overtaking dynamics while azimuthal (or equivalently axial) perturbations favour leapfrog. These observations are understood by the nonlinear dynamics of simplified inviscid models: two point-vortex alleys when $\hat{L} \lesssim 0.1$, two vortex-ring-filament alleys when $\hat{L} \lesssim 0.3$. Such dynamics are described using phase portraits of iso-Hamiltonian lines: they consist in bounded and unbounded trajectories respectively corresponding to leapfrog and overtaking. Dissipation is shown to turn overtaking into leapfrog, and is known thereafter to turn leapfrog to vortex merging.

At larger pitch, such dynamics are still observed up to $\hat{L} \approx 0.85$ if $\hat{a}_{0}=0.06$ (or $\hat{L} \approx 1$ if $\hat{a}_{0}<0.01$ ). A model of two small-core helical vortices in the cut-off approximation is then more appropriate to account for the dynamics. At that point, the amplitude of nonlinear oscillations originating from small perturbations reaches a maximum, and begins to diminish abruptly when further increasing the pitch. Small perturbations then induce different types of fluttering, while large amplitude perturbations are still able to trigger leapfrog. The system stabilizes as the pitch reaches the value $\hat{L} \approx 1.1$ if $\hat{a}_{0}=0.06$ (or $\hat{L} \approx 1.3$ if $\hat{a}_{0}<0.01$ ). These observations are corroborated qualitatively and quantitatively by the phase portraits that can be drawn using the cut-off filament approximation. Such portraits thus allow to understand most of the dynamics of the two he- 
(a)

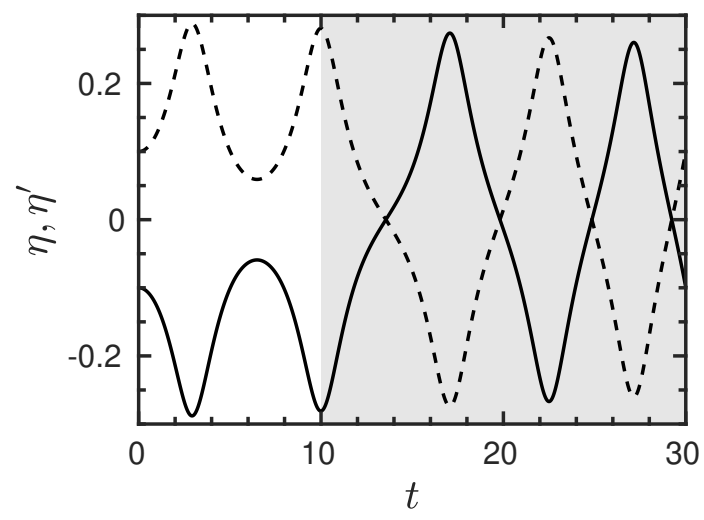

(b)

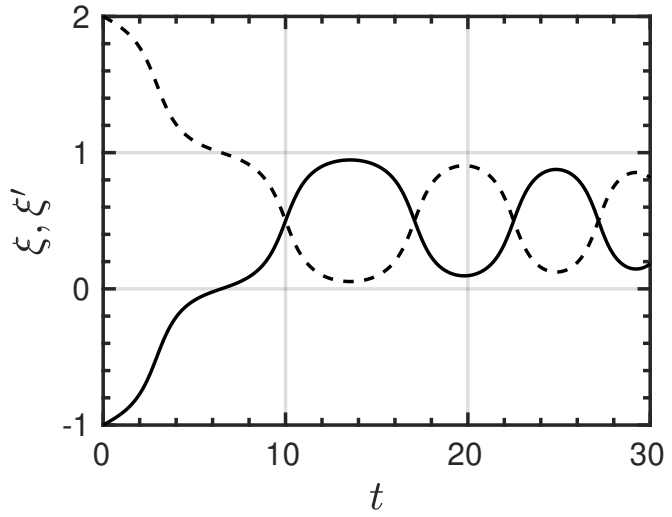

(c)

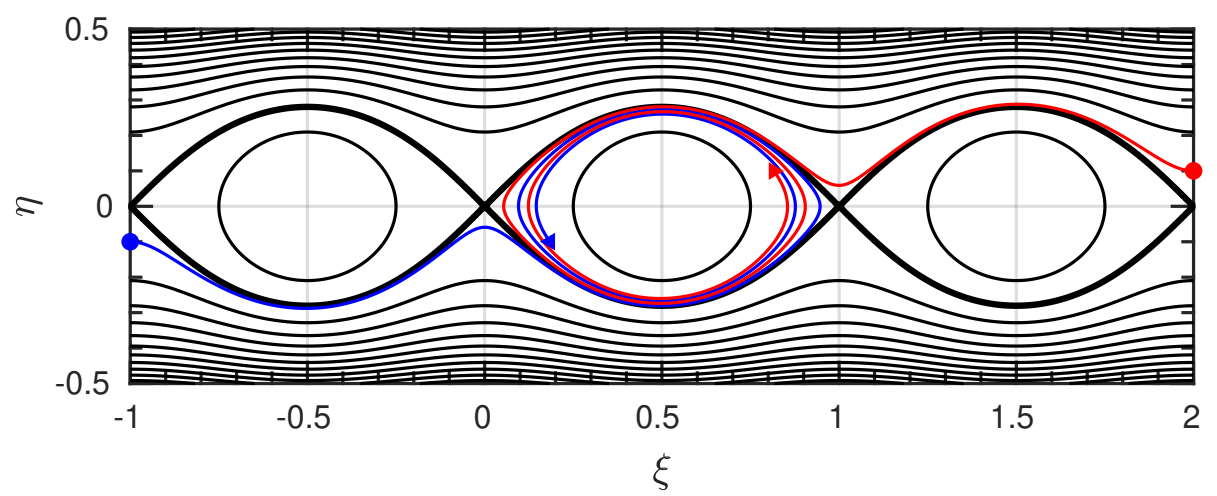

FIG. 17. Time integration of the dissipative toy model (19) with $v^{\prime}=0.01$. The initial perturbation is a transverse shift of amplitude $|\eta|=0.1$. (a) Transverse locations $\eta$ and $\eta^{\prime}$ and (b) axial locations $\xi$ and $\xi^{\prime}$ of the two vortex alleys. (c) Trajectories of the two alleys (in red and blue) in the $(\xi, \eta)$ plane. The blue and red dots correspond to the initial time.

lical vortex system over the whole range of pitches. In particular, their topology shows how different choices of initial vortex locations can induce different behaviors and how tip vortices may hence be manipulated in applications.

In a companion paper, DNS results are thoroughly compared to the inviscid predictions using phase portraits presented herein [26]. Inviscid predictions are clearly limited due to the model hypotheses: the vortex core is assumed small with respect to other typical lengths (helix pitch and radius) and remains axisymmetric. Long-time dynamics lead to significant vortex core spreading, which in turn induces partial pairing. This unavoidably affects quantitative predictions at large pitch as well as makes the merging onset inaccurate. Ongoing work is devoted to include exact higher-order core size effects and diffusion in the dynamical model. This work could also be extended to the system of three helical vortices pertinent for wind turbine wakes, although the dynamical phase space is of larger dimension. 


\section{ACKNOWLEDGMENTS}

This work received support from the French Agence Nationale de la Recherche under the ANR HELIX project ANR-12-BS09-0023-01. Simulations were run on HPC resources from GENCI-IDRIS (Grant No. 2018-2a1386) which are acknowledged, as well as from LIMSI-CNRS.

Appendix A: Dynamics of point-vortex alleys and coaxial vortex-ring alleys

Dynamics of point-vortex alleys. When a two-dimensional vortex flow is described by a set of $N_{\mathrm{v}}$ points vortices labelled by index $j=1, \ldots, N_{\mathrm{v}}$ of circulation $\Gamma_{j}$ and position $\left[x_{j}(t), y_{j}(t)\right]$, the vorticity field reads $\boldsymbol{\omega}(x, y, t)=\sum_{j=1}^{N_{\mathrm{v}}} \Gamma_{j} \delta\left(x-x_{j}\right) \boldsymbol{\delta}\left(y-y_{j}\right)$ where $\delta$ stands for the Dirac distribution. Using the complex position $z \equiv x+\mathrm{i} y$, the complex velocity field $\mathcal{U}(z, t) \equiv u_{x}(x, y, t)-\mathrm{i} u_{y}(x, y, t)$ derives from a complex potential $\Phi(z)$ obtained by Biot-Savart law

$$
\mathcal{U}(z, t)=\frac{d \Phi}{d z}=\sum_{j=1}^{N_{\mathrm{v}}} \Gamma_{j} \mathcal{K}\left(z-z_{j}\right) \text { with } \mathcal{K}\left(z^{\prime}\right) \equiv \frac{1}{2 \pi \mathrm{i} z^{\prime}} \text { and } \Phi(z)=\frac{1}{2 \pi \mathrm{i}} \sum_{j=1}^{N_{\mathrm{v}}} \Gamma_{j} \log \left(z-z_{j}\right)
$$

The real (resp. imaginary) part of $\Phi(z)$ corresponds to the potential $\phi(x, y)$ (resp. the streamfunction $\psi(x, y)$ ). In a set of point vortices, the action of a given point vortex on itself should be removed for physical reasons. This avoids a mathematical singularity [22] and provides the governing equation for vortex $i$

$$
\frac{d}{d t} z_{i}=\sum_{\substack{j=1 \\ j \neq i}}^{N_{\mathrm{v}}} \Gamma_{j} \mathcal{K}\left(z_{i}-z_{j}\right)
$$

System (A2) is Hamiltonian: if one defines $q_{i} \equiv x_{i}$ and $p_{i} \equiv \Gamma_{i} y_{i}$, it can be rewritten for $i=1, \cdots, N_{\mathrm{v}}$ as:

$$
\dot{q}_{i}=\frac{\partial H}{\partial p_{i}}, \quad \dot{p}_{i}=-\frac{\partial H}{\partial q_{i}}, \quad \text { where } H \equiv-\frac{1}{4 \pi} \sum_{i=1}^{N_{\mathrm{v}}} \sum_{j \neq i}^{N_{\mathrm{v}}} \Gamma_{i} \Gamma_{j} \log \left[\sqrt{\left(x_{i}-x_{j}\right)^{2}+\left(y_{i}-y_{j}\right)^{2}}\right],
$$

The Hamiltonian $H$ is conserved, as well as three other quantities: momentum components $P_{x}=\sum_{i=1}^{N_{\mathrm{v}}} y_{i} \Gamma_{i}$ and $P_{y}=-\sum_{i=1}^{N_{\mathrm{v}}} x_{i} \Gamma_{i}$, and angular momentum $L_{z}=\sum_{i=1}^{N_{\mathrm{v}}}\left[x_{i}^{2}+y_{i}^{2}\right] \Gamma_{i}$. Consider now the case of two periodic alleys of point vortices. Each alley labelled by an index $p$ is made of an infinite number of vortices with identical circulation $\Gamma_{p}$, located at $\left(x_{p}(t)+k \ell, y_{p}(t)\right)$ with $k \in \mathbb{Z}$. Properties valid for finite numbers of vortices can be extended: for instance, equation (A1) turns into

$$
\mathcal{U}(z, t)=\sum_{p=1,2} \Gamma_{p} \mathcal{K}_{\infty}\left(z-z_{p}\right) \text { with } \mathcal{K}_{\infty}(z)=\sum_{k=-\infty}^{+\infty} \mathcal{K}(z-k \ell)
$$

and $z_{p} \equiv x_{p}+\mathrm{i} y_{p}$. The Herglotz trick $\sum_{k=-\infty}^{+\infty}(z+k)^{-1}=\pi / \tan (\pi z)$ known in complex analysis [2] yields 
the following identities

$$
\mathcal{K}_{\infty}(z)=\frac{1}{2 \mathrm{i} \ell \tan (\pi z / \ell)}, \quad \Phi(z)=\frac{1}{2 \pi \mathrm{i}} \sum_{p=1,2} \Gamma_{p} \log \sin \left[\pi\left(z-z_{p}\right) / \ell\right],
$$

leading to the streamfunction $\psi$ defined up to an additive constant

$$
\psi(x, y)=-\frac{1}{4 \pi} \sum_{p=1,2} \Gamma_{p} \log \left\{\cosh \left[2 \pi\left(y-y_{p}\right) / \ell\right]-\cos \left[2 \pi\left(x-x_{p}\right) / \ell\right]\right\} .
$$

The infinite periodic alley number $i$ is not self-propelled: any point vortex located at $\left(x_{i}, y_{i}\right)$ is not affected by the set of other point vortices from the same alley since the action of vortex located at $\left(x_{i}+k_{1} \ell, y_{i}\right)$ with $k_{1} \in \mathbb{Z}^{\star}$ is cancelled by the action of the companion vortex located at $\left(x_{i}-k_{1} \ell, y_{i}\right)$. The infinite alley $i$ thus moves only because of the other alleys leading to the velocity

$$
\frac{d}{d t}\left(\begin{array}{l}
x_{i} \\
y_{i}
\end{array}\right)=\frac{1}{2 \ell} \sum_{\substack{p=1,2 \\
p \neq i}} \Gamma_{p} \frac{1}{\cosh \left[2 \pi\left(y_{i}-y_{p}\right) / \ell\right]-\cos \left[2 \pi\left(x_{i}-x_{p}\right) / \ell\right]}\left(\begin{array}{c}
-\sinh \left[2 \pi\left(y_{i}-y_{p}\right) / \ell\right] \\
\sin \left[2 \pi\left(x_{i}-x_{p}\right) / \ell\right]
\end{array}\right) .
$$

This finite dynamical system is also Hamiltonian: if one defines $q_{i} \equiv x_{i}$ and $p_{i} \equiv \Gamma_{i} y_{i}$, system (A7) can be rewritten for $i=1,2$ as equation (A3) but where the Hamiltonian $H$ becomes

$$
H \equiv-\frac{1}{8 \pi} \sum_{i=1,2} \sum_{\substack{j=1,2 \\ j \neq i}} \Gamma_{i} \Gamma_{j} \log S_{i j}, S_{i j} \equiv \cosh \left[2 \pi\left(y_{i}-y_{j}\right) / \ell\right]-\cos \left[2 \pi\left(x_{i}-x_{j}\right) / \ell\right] .
$$

This is a conserved quantity, as well as the two components of momentum $P_{x}=y_{1} \Gamma_{1}+y_{2} \Gamma_{2}$ and $P_{y}=$ $x_{1} \Gamma_{1}+x_{2} \Gamma_{2}$ over a periodic domain $x \in[0, \ell]$. By contrast, the angular momentum $\left[x_{1}^{2}+y_{1}^{2}\right] \Gamma_{1}+\left[x_{2}^{2}+y_{2}^{2}\right] \Gamma_{2}$ which is conserved for a finite number of vortices, is no more defined for the infinite alley [27], this latter quantity becoming infinite for an infinite row.

Dynamics of coaxial vortex-ring alleys. Let us introduce a finite number of coaxial ring filaments $i=$ $1, \ldots, N_{\mathrm{v}}$ characterized by a fixed circulation $\Gamma_{i}$, a position $z_{i}(t)$ along the $z$-axis of symmetry, a radial coordinate $r_{i}(t)$ and a core size $a_{i}(t)$. Such a system is known to admit a Hamiltonian formulation $[15,17,22]$ like equation (A3) where variable $q_{i}$ is the location $z_{i}(t)$ and its conjugated variable $p_{i}$ is the $z$-component of momentum $\Gamma_{i} \pi r_{i}^{2}$. The Hamiltonian is now the sum of two contributions $H=H_{\text {self }}+H_{\text {int }}$. Quantity $H_{\text {self }}$ corresponds to the self-energy of the rings [22]

$$
H_{\text {self }} \equiv \frac{1}{2} \sum_{i=1}^{N_{\mathrm{v}}} \Gamma_{i}^{2} r_{i}\left(\log \frac{8 r_{i}}{a_{i}}-2+C\right)
$$

where the ring core size $a_{i}(t)$ is assumed always small with respect to $r_{i}$ as well as with respect to the distance between two nearby rings. The dimensionless factor $C=\frac{1}{2}-\log \left(2 \delta_{i}\right)$ depends of the velocity profile inside the vortex core [see definitions below in (B9) or (B10)]. Generally $C$ depends slightly on the core size via the small parameter $\left(a_{i} / r_{i}\right)^{2} \log \left(8 r_{i} / a_{i}\right) \ll 1$ [22]. For a Rankine (resp. a Lamb-Oseen) profile, it equals $C=\frac{1}{4}$ (resp. $C \approx-0.05772$ ). In addition, the conservation of the vortex ring volume $2 \pi^{2} r_{i} a_{i}^{2}(t)$ imposes

$$
r_{i} a_{i}^{2}=r_{i}^{0}\left(a_{i}^{0}\right)^{2}
$$


where $r_{i}^{0}$ and $a_{i}^{0}$ stand for the reference values of radius and core size of the state from which the vortex was moved. Using the constraint (A10), $H_{\text {self }}$ reads

$$
H_{\text {self }}=\sum_{i=1}^{N_{\mathrm{v}}} \Gamma_{i}^{2} r_{i}\left(\frac{3}{4} \log r_{i}-\frac{1}{4} \log r_{i}^{0}-\frac{1}{2} \log a_{i}^{0}+F\right), \quad \text { with } F \equiv \frac{3}{2} \log 2-1+\frac{1}{2} C .
$$

The second part $H_{\text {int }}$ of the Hamiltonian corresponds to the mutual interaction between rings [15, 17, 22]

$$
H_{\mathrm{int}} \equiv \frac{\pi}{2} \sum_{i=1}^{N_{\mathrm{v}}} \sum_{\substack{j=1 \\ j \neq i}}^{N_{\mathrm{v}}} \Gamma_{i} \Gamma_{j} r_{i} r_{j} \mathscr{I}\left(r_{i}, r_{j}, z_{i}-z_{j}\right)
$$

where the integral

$$
\mathscr{I}(r, s, z)=\frac{1}{2 \pi} \int_{0}^{2 \pi} \frac{\cos \varphi \mathrm{d} \varphi}{\sqrt{z^{2}+r^{2}+s^{2}-2 r s \cos \varphi}}
$$

does not depend on the core sizes $a_{i}$ and has the symmetry properties $\mathscr{I}(r, s, z)=\mathscr{I}(r, s,-z)=\mathscr{I}(s, r, z)$. It can be expressed as

$$
\mathscr{I}(r, s, z)=\frac{1}{\pi \sqrt{r s}}\left[\left(\frac{2}{\alpha}-\alpha\right) \mathscr{K}(\alpha)-\frac{2}{\alpha} \mathscr{E}(\alpha)\right], \quad \alpha^{2}=\frac{4 r s}{z^{2}+(r+s)^{2}},
$$

where $\mathscr{K}(\alpha)$ and $\mathscr{E}(\alpha)$ are elliptical integrals of the first and second species

$$
\mathscr{K}(\alpha)=\int_{0}^{\pi / 2} \frac{\mathrm{d} \varphi}{\sqrt{1-\alpha^{2} \sin ^{2} \varphi}}, \quad \mathscr{E}(\alpha)=\int_{0}^{\pi / 2} \sqrt{1-\alpha^{2} \sin ^{2} \varphi} \mathrm{d} \varphi .
$$

Note that there are two invariants of motion: the Hamiltonian $H$ and the $z$-component of the total momentum $P_{z}=\sum_{i=1}^{N_{\mathrm{v}}} p_{i} \equiv \sum_{i=1}^{N_{\mathrm{v}}} \Gamma_{i} \pi r_{i}^{2}$ [17]. Let us now consider two alleys of coaxial vortex rings, each alley being periodic of period $\ell$ with rings of identical circulation $\Gamma$. In a similar fashion than in appendix $\mathrm{A}$, this system is a generalization of the finite number of coaxial rings when $N_{\mathrm{v}}=2 N$ with $N \rightarrow \infty$. The dynamical system can be written as a Hamiltonian $\hat{H}=\lim _{N \rightarrow \infty} H /\left(\Gamma^{2} N\right)$ which is the value over a spatial period

$$
\begin{aligned}
\hat{H}= & r_{1}\left(\frac{3}{4} \log r_{1}-\frac{1}{4} \log r_{1}^{0}-\frac{1}{2} \log a_{1}^{0}+F\right)+r_{2}\left(\frac{3}{4} \log r_{2}-\frac{1}{4} \log r_{2}^{0}-\frac{1}{2} \log a_{2}^{0}+F\right) \\
& +\pi r_{1}^{2} \sum_{k=1}^{\infty} \mathscr{I}\left(r_{1}, r_{1}, k \ell\right)+\pi r_{2}^{2} \sum_{k=1}^{\infty} \mathscr{I}\left(r_{2}, r_{2}, k \ell\right)+\pi r_{1} r_{2} \sum_{k=-\infty}^{+\infty} \mathscr{I}\left(r_{1}, r_{2}, z_{1}-z_{2}+k \ell\right) .
\end{aligned}
$$

Only three degrees of freedom intervene in this system: the radii $r_{1}$ and $r_{2}$ characteristic of each alley, as well as the relative axial position $z_{1}-z_{2}$ of the two alleys. In addition, the conservation of the $z$-component of momentum $P_{z} \equiv \Gamma \pi\left[r_{1}^{2}+r_{2}^{2}\right]$ over a periodic domain $x \in\left[0, \ell\left[\right.\right.$ imposes a relation between $r_{1}$ and $r_{2}$. The dynamical system is thus two-dimensional. 


\section{Appendix B: Velocity induced on a helical vortex in the cut-off approximation}

Let us consider two helical filaments indexed by subscript $p=1,2$ of reduced pitch $L$. The full vortex line $p$ is given by position $\boldsymbol{r}_{p}$

$$
\boldsymbol{r}_{p}(\theta, t)=r_{p}(t) \boldsymbol{e}_{r}\left(\varphi_{p}(t)+\theta\right)+\frac{\theta}{\gamma} \boldsymbol{e}_{z}, \quad \text { with } \gamma \equiv \frac{1}{L},
$$

when varying continuously the $\theta$ parameter. To comply with helical symmetry, $L$ is kept constant in time and identical for all filaments. It is adequate to introduce on each filament $p$, a Frenet-Serret basis $\left(\boldsymbol{t}_{p}, \boldsymbol{n}_{p}, \boldsymbol{b}_{p}\right)$ where $\boldsymbol{t}_{\boldsymbol{p}}$ denotes the vector tangent to the filament directed always in the direction of increasing $z$

$$
\boldsymbol{t}_{p}=\frac{1}{\sqrt{1+\gamma^{2} r_{p}^{2}}}\left[-\sin \left(\varphi_{p}+\theta\right) \gamma r_{p} \boldsymbol{e}_{x}+\cos \left(\varphi_{p}+\theta\right) \gamma r_{p} \boldsymbol{e}_{y}+\boldsymbol{e}_{z}\right],
$$

$\boldsymbol{n}_{p}=-\cos \left(\varphi_{p}+\theta\right) \boldsymbol{e}_{x}-\sin \left(\varphi_{p}+\theta\right) \boldsymbol{e}_{y}$ the unit normal vector, $\boldsymbol{b}_{p} \equiv \boldsymbol{t}_{p} \times \boldsymbol{n}_{p}$ the binormal vector.

If we determine the motion of the particular fluid particle located at time $t$ at the intersection with the plane $z=0$, we are able to recover the position and motion of the whole filament $p$ because of the helical symmetry. Let us hence compute the velocity $\boldsymbol{u}_{p}$ at that particular point $\boldsymbol{r}^{0}(t) \equiv r_{p}(t) \boldsymbol{e}_{r}\left(\boldsymbol{\varphi}_{p}(t)\right)$. It can be decomposed into two contributions: the induced motion of the filament $p$ on itself contained in $\boldsymbol{u}_{p}^{\text {self }}$ and the velocity $\boldsymbol{u}_{p}^{\text {ind }}$ induced by all other filaments (the filament labelled by the index $q$ having the contribution $\boldsymbol{u}_{p, q}^{\text {ind }}$ ):

$$
\boldsymbol{u}_{p}=\boldsymbol{u}_{p}^{\text {self }}+\boldsymbol{u}_{p}^{\text {ind }}, \quad \text { with } \boldsymbol{u}_{p}^{\text {ind }}=\sum_{q \neq p} \boldsymbol{u}_{p, q}^{\text {ind }}
$$

Velocity $\boldsymbol{u}_{p, q}^{\text {ind }}$ induced by helical vortex $q$ on vortex $p \neq q$. The velocity induced by the filament $q$ at a point $\boldsymbol{r}^{0}(t)$ away from the filament $q$ is given by the Biot-Savart expression:

$$
\frac{\Gamma_{q}}{4 \pi} \int_{-\infty}^{+\infty} \boldsymbol{t}_{q} \times \frac{\boldsymbol{r}^{0}(t)-\boldsymbol{r}_{\boldsymbol{q}}\left(s_{q}\right)}{\left\|\boldsymbol{r}^{0}(t)-\boldsymbol{r}_{\boldsymbol{q}}\left(s_{q}\right)\right\|^{3}} \mathrm{~d} s_{q}
$$

where $s_{q} \equiv\left(1+\gamma^{2} r_{q}^{2}\right)^{1 / 2} \theta / \gamma$ is the arclength along vortex filament $q$. Consider first a simpler case: the velocity induced by the vortex filament $q$ on the filament $p$ located at $\boldsymbol{r}^{0}=r_{p} \boldsymbol{e}_{x}$ i.e. $\varphi_{p}=0$ (it is assumed that $r_{p} \boldsymbol{e}_{x}$ is a point exterior to filament $q$ ). The vector product in the integrand of (B4) reads

$$
\boldsymbol{t}_{q} \times\left[r_{p} \boldsymbol{e}_{x}-\boldsymbol{r}_{q}(\theta, t)\right] \mathrm{d} s_{q}=\frac{r_{q} \mathrm{~d} \theta}{\gamma}\left(\begin{array}{c}
\sin \left(\theta+\varphi_{q}\right)-\theta \cos \left(\theta+\varphi_{q}\right) \\
r_{p} / r_{q}-\cos \left(\theta+\varphi_{q}\right)-\theta \sin \left(\theta+\varphi_{q}\right) \\
\gamma r_{q}\left[1-\left(r_{p} / r_{q}\right) \cos \left(\theta+\varphi_{q}\right)\right]
\end{array}\right)
$$

and the denominator equals

$$
\left|r_{p} \boldsymbol{e}_{x}-\boldsymbol{r}_{q}(\theta, t)\right|^{3}=\left[r_{p}^{2}+r_{q}^{2}-2 r_{q} r_{p} \cos \left(\theta+\varphi_{q}\right)+\theta^{2} / \gamma^{2}\right]^{3 / 2} .
$$


After some manipulations, the induced velocity can be written under the form

$$
\boldsymbol{u}_{p, q}^{\text {ind }}=\frac{\Gamma_{q} \gamma^{2}}{4 \pi}\left[r_{q} E_{1} \boldsymbol{e}_{x}+\left(r_{p} E_{2}-r_{q} E_{3}\right) \boldsymbol{e}_{y}+\left(\gamma r_{q}^{2} E_{2}-\gamma r_{p} r_{q} E_{4}\right) \boldsymbol{e}_{z}\right]
$$

where

$$
\begin{aligned}
& E_{1}=\int_{-\infty}^{+\infty} \frac{\sin \left(\theta+\varphi_{q}\right)-\theta \cos \left(\theta+\varphi_{q}\right)}{\left[\gamma^{2} r_{p}^{2}+\gamma^{2} r_{q}^{2}-2 \gamma^{2} r_{q} r_{p} \cos \left(\theta+\varphi_{q}\right)+\theta^{2}\right]^{3 / 2}} \mathrm{~d} \theta \\
& E_{2}=\int_{-\infty}^{+\infty} \frac{1}{\left[\gamma^{2} r_{p}^{2}+\gamma^{2} r_{q}^{2}-2 \gamma^{2} r_{q} r_{p} \cos \left(\theta+\varphi_{q}\right)+\theta^{2}\right]^{3 / 2}} \mathrm{~d} \theta \\
& E_{3}=\int_{-\infty}^{+\infty} \frac{\cos \left(\theta+\varphi_{q}\right)+\theta \sin \left(\theta+\varphi_{q}\right)}{\left[\gamma^{2} r_{p}^{2}+\gamma^{2} r_{q}^{2}-2 \gamma^{2} r_{q} r_{p} \cos \left(\theta+\varphi_{q}\right)+\theta^{2}\right]^{3 / 2}} \mathrm{~d} \theta \\
& E_{4}=\int_{-\infty}^{+\infty} \frac{\cos \left(\theta+\varphi_{q}\right)}{\left[\gamma^{2} r_{p}^{2}+\gamma^{2} r_{q}^{2}-2 \gamma^{2} r_{q} r_{p} \cos \left(\theta+\varphi_{q}\right)+\theta^{2}\right]^{3 / 2}} \mathrm{~d} \theta
\end{aligned}
$$

are dimensionless quantities. In order to obtain the velocity induced by a helical vortex $q$ on vortex $p \neq q$ located at $r_{p}(t) \boldsymbol{e}_{r}\left(\varphi_{p}(t)\right)$, one generalizes the above computation by changing axes, transforming phase $\varphi_{q}$ to $\varphi_{q}-\varphi_{p}$ :

$$
\boldsymbol{u}_{p, q}^{\text {ind }}=\frac{\Gamma_{q} \gamma^{2}}{4 \pi}\left[r_{q} E_{1}^{\prime} \boldsymbol{e}_{r}\left(\varphi_{p}\right)+\left(r_{p} E_{2}^{\prime}-r_{q} E_{3}^{\prime}\right) \boldsymbol{e}_{\theta}\left(\varphi_{p}\right)+\left(\gamma r_{q}^{2} E_{2}^{\prime}-\gamma r_{p} r_{q} E_{4}^{\prime}\right) \boldsymbol{e}_{z}\right]
$$

where $E_{j}^{\prime}$ is obtained from expression $E_{j}$ by transforming $\varphi_{q}$ into $\varphi_{q}-\varphi_{p}$. By using trigonometric relations, one obtains

$$
\begin{gathered}
E_{1}^{\prime}=A_{p, q} \cos \left(\varphi_{q}-\varphi_{p}\right)+B_{p, q} \sin \left(\varphi_{q}-\varphi_{p}\right), \quad E_{2}^{\prime}=F_{p, q}, \\
E_{3}^{\prime}=-A_{p, q} \sin \left(\varphi_{q}-\varphi_{p}\right)+B_{p, q} \cos \left(\varphi_{q}-\varphi_{p}\right), \quad E_{4}^{\prime}=C_{p, q} \cos \left(\varphi_{q}-\varphi_{p}\right)-D_{p, q} \sin \left(\varphi_{q}-\varphi_{p}\right),
\end{gathered}
$$

where

$$
\begin{aligned}
& A_{p, q}=\int_{-\infty}^{+\infty}[\sin (\theta)-\theta \cos (\theta)] Y_{p, q} \mathrm{~d} \theta, \quad B_{p, q}=\int_{-\infty}^{+\infty}[\cos (\theta)+\theta \sin (\theta)] Y_{p, q} \mathrm{~d} \theta, \\
& C_{p, q}=\int_{-\infty}^{+\infty} \cos (\theta) Y_{p, q} \mathrm{~d} \theta, \quad D_{p, q}=\int_{-\infty}^{+\infty} \sin (\theta) Y_{p, q} \mathrm{~d} \theta, \quad F_{p, q}=\int_{-\infty}^{+\infty} Y_{p, q} \mathrm{~d} \theta, \\
& Y_{p, q}=\frac{1}{Z_{p, q}^{3 / 2}}, \quad Z_{p, q}=\theta^{2}+\gamma^{2} r_{p}^{2}+\gamma^{2} r_{q}^{2}-2 \gamma^{2} r_{q} r_{p} \cos \theta \cos \left(\varphi_{q}-\varphi_{p}\right)+2 \gamma^{2} r_{q} r_{p} \sin \theta \sin \left(\varphi_{q}-\varphi_{p}\right)
\end{aligned}
$$

are dimensionless quantities which depend on $r_{p}, r_{q}$ and $\varphi_{q}-\varphi_{p}$. The following (anti-)symmetries hold

$$
B_{p, q}=B_{q, p}, \quad C_{p, q}=C_{q, p}, \quad F_{p, q}=F_{q, p}, \quad A_{p, q}=-A_{q, p}, \quad D_{p, q}=-D_{q, p} .
$$

The conservation of $\Gamma\left(r_{1}^{2}+r_{2}^{2}\right)$ in the case of 2 vortices precisely occurs because $B_{1,2}=B_{2,1}$ and $A_{1,2}=$ $-A_{2,1}$. In addition, when $\varphi_{q}-\varphi_{p}=\pi$, one has additionally $A_{p, q}=A_{q, p}$ and $D_{p, q}=D_{q, p}$, hence $A_{p, q}=$ $D_{p, q}=0$. 
Self-induced velocity $\boldsymbol{u}_{p}^{\text {self }}$ of helical vortex $p$. Let us now compute the self-induced velocity $\boldsymbol{u}_{p}^{\text {self }}$ of filament $p$ at a point $r^{0}(t)$. First we consider again the simpler case in which the vortex filament $p$ is such that $\varphi_{p}=0$ so that $\boldsymbol{r}^{0}(t)=r_{p}\left(s_{p}=0\right) \boldsymbol{e}_{x}$. The self-induced velocity at that point is given by the Biot-Savart law. The integral however is singular and should be regularized using the cut-off theory, in which the vortex core size $a_{p}(t)$ is introduced. More precisely the cut-off theory gives the velocity at point $r_{p}\left(s_{p}=0\right) \boldsymbol{e}_{x}$ under the form

$$
\boldsymbol{u}_{p}^{\mathrm{self}}=\frac{\Gamma_{p}}{4 \pi} \mathcal{F}_{-\infty}^{+\infty} \boldsymbol{t}_{\boldsymbol{p}} \times \frac{r_{p}(0) \boldsymbol{e}_{x}-\boldsymbol{r}_{\boldsymbol{p}}\left(s_{p}\right)}{\left\|r_{p}(0) \boldsymbol{e}_{x}-\boldsymbol{r}_{\boldsymbol{p}}\left(s_{p}\right)\right\|^{3}} \mathrm{~d} s_{p}
$$

where $s_{p} \equiv \theta\left(1+\gamma^{2} r_{p}^{2}\right)^{1 / 2} / \gamma$ is the arclength along vortex filament $p$, with the origin at the intersection with the plane $z=0$. The cross on the integral means that the cut-off theory removes from the integral domain $\left.s_{p} \in\right]-\infty, \infty[$ an interval $]-\delta_{p} a_{p}, \delta_{p} a_{p}$ [ around the point at which the velocity is evaluated (here at $s_{p}=0$ ). Quantity $\delta_{p}$ is a dimensionless factor depending on the velocity profile inside the vortex core. For a compact vorticity field included in a disk of radius $a_{p}$

$$
\ln \left(2 \delta_{p}\right)=\frac{1}{2}+\frac{8 \pi^{2}}{\Gamma^{2}} \int_{0}^{a_{p}} r^{\prime}\left[u^{2}\left(r^{\prime}\right)-\frac{1}{2} w^{2}\left(r^{\prime}\right)\right] \mathrm{d} r^{\prime}
$$

where $r^{\prime}$ denotes the local radial coordinate of the axisymmetric vortex filament and $w\left(r^{\prime}\right)$ [resp. $u\left(r^{\prime}\right)$ ] is the local azimuthal (resp. axial) velocity profile. If the vorticity field is not compact but exponentially small as $r^{\prime} \rightarrow \infty$, then

$$
\ln \left(2 \delta_{p}\right)=\frac{1}{2}+\lim _{r \rightarrow+\infty}\left[\log \left(\frac{r}{a_{p}}\right)-\frac{4 \pi^{2}}{\Gamma^{2}} \int_{0}^{r} r^{\prime} w^{2}\left(r^{\prime}\right) \mathrm{d} r^{\prime}\right]+\frac{8 \pi^{2}}{\Gamma^{2}} \int_{0}^{\infty} r^{\prime} u^{2}\left(r^{\prime}\right) \mathrm{d} r^{\prime} .
$$

For instance, this formula yields $\delta_{p} \approx 0.8735$ for a Gaussian vortex.

The vector product in the integrand of (B8) reads

$$
\boldsymbol{t}_{p} \times\left[r_{p} \boldsymbol{e}_{x}-\boldsymbol{r}_{p}(\theta, t)\right] \mathrm{d} s_{p}=\frac{r_{p} \mathrm{~d} \theta}{\gamma}\left(\begin{array}{c}
\sin (\theta)-\theta \cos (\theta) \\
1-\cos (\theta)-\theta \sin (\theta) \\
\gamma r_{p}[1-\cos (\theta)]
\end{array}\right),
$$

and the denominator equals

$$
\left|r_{p} \boldsymbol{e}_{x}-\boldsymbol{r}_{p}(\theta, t)\right|^{3}=\left[r_{p}^{2}+r_{q}^{2}-2 r_{q} r_{p} \cos \theta+\theta^{2} / \gamma^{2}\right]^{3 / 2} .
$$

The self-induced velocity $\boldsymbol{u}_{p}^{\text {self }}$ then reads

$$
\boldsymbol{u}_{p}^{\mathrm{self}}=\frac{\Gamma_{p} r_{p} \gamma^{2}}{2 \pi}\left\{\left[\mathscr{G}_{1}\left(\gamma r_{p},|\gamma| \delta_{p} a_{p}\right)-\mathscr{G}_{2}\left(\gamma r_{p},|\gamma| \delta_{p} a_{p}\right)\right] \boldsymbol{e}_{y}+\gamma r_{p} \mathscr{G}_{2}\left(\gamma r_{p},|\gamma| \delta_{p} a_{p}\right) \boldsymbol{e}_{z}\right\}
$$

with

$$
\mathscr{G}_{1}(A, B)=\frac{1}{A^{2}}\left\{1-\frac{\theta_{0}}{\left[2 A^{2}\left(1-\cos \theta_{0}\right)+\theta_{0}^{2}\right]^{1 / 2}}\right\}, \quad \mathscr{G}_{2}(A, B) \equiv \int_{\theta_{0}}^{\infty} \frac{(1-\cos \theta) \mathrm{d} \theta}{\left[\theta^{2}+2 A^{2}(1-\cos \theta)\right]^{3 / 2}},
$$

and $\theta_{0}(A, B) \equiv B / \sqrt{1+A^{2}}$. 
The cut-off theory thus removes an interval $]-\theta_{0 p}, \theta_{0 p}[$ from the above integrals. By an adequate rotation, one eventually deduces the self-induced velocity $\boldsymbol{u}_{p}^{\text {self }}$ of filament $p$ at point $\boldsymbol{r}^{0}=r_{p}(t) \boldsymbol{e}_{r}\left(\varphi_{p}(t)\right)$ :

$$
\boldsymbol{u}_{p}^{\text {self }}=\frac{\Gamma r_{p} \gamma^{2}}{2 \pi}\left\{\left[\mathscr{G}_{1}\left(\gamma r_{p},|\gamma| \delta_{p} a_{p}\right)-\mathscr{G}_{2}\left(\gamma r_{p},|\gamma| \delta_{p} a_{p}\right)\right] \boldsymbol{e}_{\theta}\left(\varphi_{p}\right)+\gamma r_{p} \mathscr{G}_{2}\left(\gamma r_{p},|\gamma| \delta_{p} a_{p}\right) \boldsymbol{e}_{z}\right\}
$$

Kinematics of a fluid particle on helical vortex $p$. Let us now consider the kinematics of any fluid particle on filament $p$, labelled by the value $\theta=\theta_{\mathrm{f}}(t)$. Such particle is located at

$$
\boldsymbol{r}_{\mathrm{f}}(t) \equiv \boldsymbol{r}_{p}\left(\theta_{\mathrm{f}}(t), t\right)=r_{p}(t) \boldsymbol{e}_{r}\left(\theta_{\mathrm{f}}(t)+\varphi_{p}(t)\right)+\frac{\theta_{\mathrm{f}}(t)}{\gamma} \boldsymbol{e}_{z} .
$$

Its Lagrangian velocity reads

$$
\frac{\mathrm{D} \boldsymbol{r}_{\mathrm{f}}}{\mathrm{D} t}=\frac{\mathrm{d} \theta_{\mathrm{f}}}{\mathrm{d} t} \frac{\partial \boldsymbol{r}_{\mathrm{f}}}{\partial \theta}+\frac{\mathrm{d} \varphi_{p}}{\mathrm{~d} t} \frac{\partial \boldsymbol{r}_{\mathrm{f}}}{\partial \varphi_{p}}+\frac{\mathrm{d} r_{p}}{\mathrm{~d} t} \frac{\partial \boldsymbol{r}_{\mathrm{f}}}{\partial r_{p}}
$$

with

$$
\frac{\partial \boldsymbol{r}_{\mathrm{f}}}{\partial \theta}=\frac{\left(1+\gamma^{2} r_{p}^{2}\right)^{1 / 2}}{\gamma} \boldsymbol{t}_{p}, \quad \frac{\partial \boldsymbol{r}_{\mathrm{f}}}{\partial \varphi_{p}}=r_{p} \boldsymbol{e}_{\theta}\left(\theta_{\mathrm{f}}+\varphi_{p}\right), \quad \frac{\partial \boldsymbol{r}_{\mathrm{f}}}{\partial r_{p}}=\boldsymbol{e}_{r}\left(\theta_{\mathrm{f}}+\varphi_{p}\right)
$$

Within the framework of filament theory, only the velocity components orthogonal to the filament can be computed for velocity at a point located on the vortex itself. This means that for the particle located at $\boldsymbol{r}^{0}(t)$ at time $t$, for which $\theta_{\mathrm{f}}(t)=0$, the condition $\mathrm{D} \boldsymbol{r}_{\mathrm{f}} / \mathrm{D} t=\boldsymbol{u}_{p}$ is imposed along $\boldsymbol{n}_{p}$ and $\boldsymbol{b}_{p}$, i.e.

$$
\begin{aligned}
& \left(\boldsymbol{u}_{p}-\frac{\mathrm{D} \boldsymbol{r}_{\mathrm{f}}}{\mathrm{D} t}\right) \cdot \boldsymbol{n}_{p}=\left[\boldsymbol{u}^{\text {self }}+\boldsymbol{u}^{\text {ind }}-r_{p} \frac{\mathrm{d} \varphi_{p}}{\mathrm{~d} t} \boldsymbol{e}_{\theta}\left(\varphi_{p}\right)-\frac{\mathrm{d} r_{p}}{\mathrm{~d} t} \boldsymbol{e}_{r}\left(\varphi_{p}\right)\right] \cdot \boldsymbol{n}_{p}=0 \\
& \left(\boldsymbol{u}_{p}-\frac{D \boldsymbol{r}_{\mathrm{f}}}{D t}\right) \cdot \boldsymbol{b}_{p}=\left[\boldsymbol{u}^{\text {self }}+\boldsymbol{u}^{\text {ind }}-r_{p} \frac{\mathrm{d} \varphi_{p}}{\mathrm{~d} t} \boldsymbol{e}_{\theta}\left(\varphi_{p}\right)-\frac{\mathrm{d} r_{p}}{\mathrm{~d} t} \boldsymbol{e}_{r}\left(\varphi_{p}\right)\right] \cdot \boldsymbol{b}_{p}=0
\end{aligned}
$$

with

$$
\boldsymbol{n}_{p}=-\boldsymbol{e}_{r}\left(\varphi_{p}\right), \quad \boldsymbol{b}_{p}=\frac{1}{\sqrt{1+\gamma^{2} r_{p}^{2}}}\left[-\boldsymbol{e}_{\theta}\left(\varphi_{p}\right)+\gamma r_{p} \boldsymbol{e}_{z}\right]
$$

Finally, the following equations are obtained:

$$
\begin{aligned}
\frac{\mathrm{d} r_{p}}{\mathrm{~d} t} & =\left(\boldsymbol{u}_{p}^{\text {self }}+\boldsymbol{u}_{p}^{\text {ind }}\right) \cdot \boldsymbol{e}_{r}\left(\boldsymbol{\varphi}_{p}\right) \\
\frac{\mathrm{d} \varphi_{p}}{\mathrm{~d} t} & =\left(\boldsymbol{u}_{p}^{\text {self }}+\boldsymbol{u}_{p}^{\text {ind }}\right) \cdot\left\{\frac{\boldsymbol{e}_{\theta}\left(\varphi_{p}\right)}{r_{p}}-\gamma \boldsymbol{e}_{z}\right\} .
\end{aligned}
$$


[1] See Supplemental Material for the movies corresponding to the vortex dynamics of Figs. 4a-b, 4c-d, 6b (axial or radial perturbations), $8 \mathrm{c}, 8 \mathrm{f}, 11 \mathrm{c}, 11 \mathrm{f}, 12 \mathrm{c}, 13 \mathrm{c}, 13 \mathrm{f}, 14 \mathrm{c}$.

[2] M. Aigner and G. M. Ziegler. Proofs from THE BOOK. Springer Verlag, 2010. doi:10.1007/978-3-642-00856-6.

[3] H. Aref. On the equilibrium and stability of a row of point vortices. J. Fluid Mech., 290:167181, 1995. doi: $10.1017 /$ S002211209500245X.

[4] D. Baldacchino and G. J. W. van Bussel. Wind turbine wake stability investigations using a vortex ring modelling approach. Journal of Physics: Conference Series, 555:012111, dec 2014. doi:10.1088/1742-6596/555/1/012111. URL https: / / doi.org/10.10882F1742-6596\%2F555\%2F12F012111.

[5] M. J. Bhagwat and J. G. Leishman. Stability analysis of helicopter rotor wakes in axial flight. Journal of the American Helicopter Society, 45(3):165-178, 2000. ISSN 2161-6027. doi:doi:10.4050/JAHS.45.165. URL https : //www. ingentaconnect. com/content/ahs/jahs/2000/00000045/00000003/art00003.

[6] H. Bolnot, S. Le Dizès, and T. Leweke. Spatio-temporal development of the pairing instability in an infinite array of vortex rings. Fluid Dynamics Research, 46(6):061405, oct 2014. doi:10.1088/0169-5983/46/6/061405. URL https://doi.org/10.1088\%2F0169-59832F 46\%2F6\%2F061405.

[7] M. Brynjell-Rahkola and D. S. Henningson. Numerical realization of helical vortices: application to vortex instability. Theoret. Comput. Fluid Dynamics, 34:120, 2020.

[8] M. Cheng, J. Lou, and T. T. Lim. Leapfrogging of multiple axial viscous vortex rings. Phys.Fluids, 27:031702, 2015.

[9] S. C. Crow. Stability theory for a pair of trailing vortices. AIAA Journal, 8(12):2172-2179, 1970. doi: 10.2514/3.6083. URL https: //doi.org/10.2514/3.6083.

[10] I. Delbende, M. Rossi, and O. Daube. Dns of flows with helical symmetry. Theoret. Comput. Fluid Dynamics, 26(1):141-160, 2012. ISSN 1432-2250. doi:10.1007/s00162-011-0241-y. URL http://dx.doi.org/10. 1007 /s00162-011-0241-y.

[11] I. Delbende, B. Piton, and M. Rossi. Merging of two helical vortices. Eur. J. Mech. B/Fluids, 49, Part B:363-372, Jan. 2015. ISSN 0997-7546. doi:10.1016/j.euromechflu.2014.04.005. URL http: / /www. sciencedirect. $\mathrm{com} / \mathrm{science/article/pii/s0997754614000570.}$

[12] M. Felli, R. Camussi, and F. Di Felice. Mechanisms of evolution of the propeller wake in the transition and far fields. J. Fluid Mech., 682:5-53, 2011. URL http://journals.cambridge.org/abstract_ S0022112011001509.

[13] H. Lamb. Hydrodynamics. Cambridge university press, 1932.

[14] H. Levy and A. G. Forsdyke. The Stability of an Infinite System of Circular Vortices. Proceedings of the Royal Society of London. Series A, Containing Papers of a Mathematical and Physical Character, 114(768):594-604, 1927. ISSN 0950-1207. URL http://www. jstor.org/stable/94831.

[15] V. V. Meleshko. Coaxial axisymmetric vortex rings: 150 years after Helmholtz. Theoret. Comput. Fluid Dynamics, 24:403-431, 2010.

[16] A. Nemes, D. Lo Jacono, H. M. Blackburn, and J. Sheridan. Mutual inductance of two helical vortices. Journal of Fluid Mechanics, 774:298310, 2015. doi:10.1017/jfm.2015.288.

[17] E. Novikov. Hamiltonian description of axisymmetric vortex flows and the system of vortex rings. Phys. Fluids, 28:2921-2922, 1985.

[18] V. L. Okulov. On the stability of multiple helical vortices. J. Fluid Mech., 521:319-342, 2004.

[19] V. L. Okulov and J. N. Sørensen. Stability of helical tip vortices in a rotor far wake. J. Fluid Mech., 576:1-25, 2007.

[20] H. U. Quaranta, H. Bolnot, and T. Leweke. Long-wave instability of a helical vortex. J. Fluid Mech., 780:687-716, 2015. URL http://journals.cambridge.org/abstract_S0022112015004796.

[21] H. U. Quaranta, M. Brynjell-Rahkola, T. Leweke, and D. S. Henningson. Local and global pairing instabilities of two interlaced helical vortices. J. Fluid Mech., 863:927955, 2019. doi:10.1017/jfm.2018.904.

[22] P. G. Saffman. Vortex Dynamics. Cambridge University Press, 1992. ISBN 9780511624063. URL http: //dx.doi.org/10.1017/CB09780511624063. Cambridge Books Online.

[23] S. Sarmast, R. Dadfar, R. F. Mikkelsen, P. Schlatter, S. Ivanell, J. Sørensen, and D. Henningson. Mutual in- 
ductance instability of the tip vortices behind a wind turbine. Journal of Fluid Mechanics, 755:705731, 2014. doi:10.1017/jfm.2014.326.

[24] C. Selçuk, I. Delbende, and M. Rossi. Helical vortices: quasi-equilibrium states and their time evolution. Phys. Rev. Fluids, 2-8:011411, 2017. doi:10.1103/PhysRevFluids.2.084701. URL https : / / I ink . aps .org/doi / $10.1103 /$ PhysRevFluids.2.084701.

[25] C. Selçuk, I. Delbende, and M. Rossi. Helical vortices: linear stability analysis and nonlinear dynamics. Fluid Dyn. Res., 50:011411, 2018.

[26] C. Selçuk, I. Delbende, and M. Rossi. Nonlinear dynamics of two helical vortices: Direct numerical simulation. In préparation, 2021.

[27] M. Stremler. On relative equilibria and integrable dynamics of point vortices in periodic domains. Theoret. Comput. Fluid Dynamics, 24:25-37, 2010.

[28] J. H. Walther, M. Gunot, E. Machefaux, J. T. Rasmussen, P. Chatelain, V. L. Okulov, J. N. Sørensen, M. Bergdorf, and P. Koumoutsakos. A numerical study of the stabilitiy of helical vortices using vortex methods. Journal of Physics: Conference Series, 75:012034, jul 2007. doi:10.1088/1742-6596/75/1/012034. URL https://doi . org/10.1088\%2F1742-6596\%2F 75\%2F12F012034.

[29] S. E. Widnall. The stability of a helical vortex filament. J. Fluid Mech., 54:641-663, 1972. ISSN 1469-7645. 